\title{
WEATHER ON OTHER WORLDS. I. DETECTION OF PERIODIC VARIABILITY IN THE L3 DWARF DENIS-P J1058.7-1548 WITH PRECISE MULTI-WAVELENGTH PHOTOMETRY
}

\author{
Aren N. Heinze ${ }^{1}$, Stanimir Metchev $^{1}$, Daniel Apai ${ }^{2,3}$, Davin Flateau $^{2,3}$, Radostin Kurtev $^{4}$, Mark Marley $^{5}$, \\ Jacqueline Radigan $^{6}$, Adam J. Burgasser ${ }^{7}$, Étienne Artigau ${ }^{8}$, And Peter Plavchan ${ }^{9}$ \\ ${ }^{1}$ Department of Physics and Astronomy, State University of New York, Stony Brook, NY 11794-3800, USA; \\ aren.heinze@stonybrook.edu, stanimir.metchev@stonybrook.edu \\ ${ }^{2}$ University of Arizona Department of Astronomy, 933 N. Cherry Avenue, Tucson, AZ 85721, USA \\ ${ }^{3}$ University of Arizona Department of Planetary Sciences and Lunar and Planetary Laboratory, 1629 E University Blvd, Tucson, AZ 85721, USA \\ ${ }^{4}$ Departamento de Fisica y Astronomia, Facultad de Ciencias, Universidad de Valparaiso, Av. Gran Bretana 1111, Casilla 5030, Valparaiso, Chile \\ ${ }^{5}$ NASA Ames Research Center, MS-245-3, Moffett Field, CA 94035, USA \\ ${ }^{6}$ Department of Astronomy and Astrophysics, University of Toronto, 50 St. George Street, Toronto, ON M5S 3H4, Canada \\ ${ }^{7}$ University of California San Diego, Center for Astrophysics and Space Science, 9500 Gilman Drive, Mail Code 0424, La Jolla, CA 92093, USA \\ ${ }^{8}$ Département de Physique and Observatoire du Mont Mégantic, Université de Montréal, C.P. 6128, Succ. Centre-Ville, Montréal, QC, H3C 3J7, Canada \\ ${ }^{9}$ NASA Exoplanet Science Institute, California Institute of Technology, M/C 100-22, 770 South Wilson Avenue, Pasadena, CA 91125, USA \\ Received 2012 December 19; accepted 2013 March 11; published 2013 April 8
}

\begin{abstract}
Photometric monitoring from warm Spitzer reveals that the L3 dwarf DENIS-P J1058.7-1548 varies sinusoidally in brightness with a period of $4.25_{-0.16}^{+0.26} \mathrm{hr}$ and an amplitude of $0.388 \% \pm 0.043 \%$ (peak-to-valley) in the $3.6 \mu \mathrm{m}$ band, confirming the reality of a $4.31 \pm 0.31 \mathrm{hr}$ periodicity detected in $J$-band photometry from the SOAR telescope. The $J$-band variations are a factor of $2.17 \pm 0.35$ larger in amplitude than those at $3.6 \mu \mathrm{m}$, while $4.5 \mu \mathrm{m}$ Spitzer observations yield a $4.5 \mu \mathrm{m} / 3.6 \mu \mathrm{m}$ amplitude ratio of only $0.23 \pm 0.15$, consistent with zero $4.5 \mu \mathrm{m}$ variability. This wide range in amplitudes indicates rotationally modulated variability due to magnetic phenomena and/or inhomogeneous cloud cover. Weak $\mathrm{H} \alpha$ emission indicates some magnetic activity, but it is difficult to explain the observed amplitudes by magnetic phenomena unless they are combined with cloud inhomogeneities (which might have a magnetic cause). However, inhomogeneous cloud cover alone can explain all our observations, and our data align with theory in requiring that the regions with the thickest clouds also have the lowest effective temperature. Combined with published $v \sin (i)$ results, our rotation period yields a 95\% confidence lower limit of $R_{*} \geqslant 0.111 R_{\odot}$, suggesting upper limits of $320 \mathrm{Myr}$ and $0.055 M_{\odot}$ on the age and mass. These limits should be regarded cautiously because of $\sim 3 \sigma$ inconsistencies with other data; however, a lower limit of $45^{\circ}$ on the inclination is more secure. DENIS-P J1058.7-1548 is only the first of nearly two dozen low-amplitude variables discovered and analyzed by the Weather on Other Worlds project.
\end{abstract}

Key words: brown dwarfs - stars: individual (DENIS-P J1058.7-1548) - stars: low-mass - stars: rotation starspots - stars: variables: general - techniques: photometric

Online-only material: color figures

\section{INTRODUCTION}

\subsection{Dwarfs, Clouds, and the L/T Transition}

The L dwarfs (Kirkpatrick et al. 1999) include brown dwarfs with ages from tens of Myr to several Gyr (depending on mass) and also the coolest and lowest mass main sequence stars (Burrows et al. 1997, 2006). Together with the T and $\mathrm{Y}$ dwarfs and the latest $\mathrm{M}$ dwarfs, they make up the ultracool dwarfs (UCDs), which have atmospheric temperatures low enough for the formation of condensates. In $\mathrm{L}$ dwarfs these take the form of thick clouds of refractory silicate "dust" and liquid iron droplets, which profoundly influence the emitted spectra (Allard et al. 2001; Ackerman \& Marley 2001). The presence of numerous condensing molecular species makes modeling these objects a complex task (Tsuji et al. 1996; Allard et al. 1997, 2001; Ackerman \& Marley 2001; Burrows et al. 2006; Helling et al. 2008; Saumon \& Marley 2008; Marley et al. 2010), but L dwarf clouds also hold the potential for fascinating phenomena such as molten iron "rain," hot silicate "snow" (see for example Ackerman \& Marley 2001), and detectable weather patterns analogous to Jupiter's Great Red Spot (Gelino 2002; Artigau et al. 2009). L dwarfs may also allow us to study young giant planets by analogy, given their similar values of effective temperature $\left(T_{\text {eff }}\right)$.

The silicate and iron clouds of $\mathrm{L}$ dwarfs form a global overcast near or above the photosphere. They redden the emitted spectrum (Allard et al. 2001; Ackerman \& Marley 2001; Knapp et al. 2004), and apparently produce the 9-11 $\mu \mathrm{m}$ absorption attributed to silicate grains in Spitzer/IRS spectra of L dwarfs (Cushing et al. 2006; Burgasser et al. 2008; Looper et al. 2008). As objects become cooler toward the L-T transition, the effects of the iron and silicate clouds diminish, resulting in bluer near-infrared (near-IR) colors. This blueward shift happens over a very small range in $T_{\text {eff }}$, and over this range the $J$-band luminosity actually increases with decreasing temperature (Dahn et al. 2002; Tinney et al. 2003; Vrba et al. 2004; see also Knapp et al. 2004). This cannot be explained by the clouds simply sinking below the photosphere with decreasing $T_{\text {eff }}$ (Burgasser et al. 2002; Burrows et al. 2006). The clouds must additionally break up (Ackerman \& Marley 2001; Burgasser et al. 2002), and/or rain out (Ackerman \& Marley 2001) as $T_{\text {eff }}$ decreases. This suggests that $\mathrm{L} / \mathrm{T}$ transition objects may have patchy clouds.

Patchy clouds on UCDs should cause rotationally modulated photometric variability due to flux differences between the 
most-cloudy and least-cloudy hemispheres. Numerous searches for variability in $\mathrm{L}$ and $\mathrm{T}$ dwarfs have been performed to date. ${ }^{10}$ The first two objects found with large (5\%-30\%) amplitude periodic variability were indeed at the $\mathrm{L} / \mathrm{T}$ transition: the $\mathrm{T} 2.5$ dwarf SIMP J013656.57+093347.3 (Artigau et al. 2009; Apai et al. 2013) and the T1.5 dwarf 2MASS J21392676+0220226 (Radigan et al. 2012; Apai et al. 2013).

However, variability at lower amplitudes has been seen in L dwarfs that, like DENIS-P J1058.7-1548 (hereafter DENIS 1058), are far from the L/T transition (Clarke et al. 2002b; Gelino et al. 2002; Koen 2004; Lane et al. 2007). Magnetic starspots are considered as a possible cause of variations by Clarke et al. (2002b) and Lane et al. (2007), with the latter preferring this explanation. Magnetic phenomena (albeit emission regions rather than dark starspots) certainly are the cause of radio and $\mathrm{H} \alpha$ variations detected in some late-M and $\mathrm{L}$ dwarfs (Hallinan et al. 2007; Berger et al. 2008, 2009). Magnetic effects could also cause the continuum variability observed in DENIS 1058 and in previous studies of L dwarfs. However, uneven clouds on a less extreme scale than is seen in $\mathrm{L} / \mathrm{T}$ transition objects would also be consistent with all the data, especially if the inhomogeneities took the form of variations in cloud thickness rather than complete clearings in the global overcast. When L dwarf spectra are fit by models including clouds with thickness parameterized by the sedimentation or "rain" parameter $f_{\text {sed }}$, introduced by Ackerman \& Marley (2001) and used widely since (Burgasser et al. 2002; Knapp et al. 2004; Saumon \& Marley 2008), it is found that different objects are best fit by different values of $f_{\text {sed }}$ (Burgasser et al. 2008; Stephens et al. 2009). Similarly, there is no reason to assume that the global clouds of a given L dwarf will be specified by a spatially and temporally unvarying value of $f_{\text {sed }}$.

The photometric effects of inhomogeneous clouds in L and $\mathrm{T}$ dwarfs depend strongly on the wavelength of observation. This is due to the strong molecular gas absorption in the atmospheres (from $\mathrm{H}_{2} \mathrm{O}$ and $\mathrm{CH}_{4}$ ), which causes the effective altitude (and thus temperature) of the photosphere to vary from one wavelength to another (Burrows et al. 1997; Ackerman \& Marley 2001; Kirkpatrick 2005; Burrows et al. 2006). This wavelength dependence makes multi-band photometric monitoring a useful tool for understanding the vertical structure of clouds in UCDs.

\subsection{The L3 Dwarf DENIS-P J1058.7-1548}

Herein we report observations of DENIS 1058 made as part of the Weather on Other Worlds (WoW) project, a Spitzer Exploration Science program probing the photometric variability of brown dwarfs (S. Metchev et al., in preparation). DENIS 1058 is the first WoW target for which we acquired ground-based photometry nearly simultaneous with the Spitzer observations.

DENIS 1058 (Delfosse et al. 1997) is an L3 dwarf (Kirkpatrick et al. 1999) at a distance of $17.33 \pm 0.30 \mathrm{pc}$ (Dahn et al. 2002). At $J=14.155 \pm 0.035 \mathrm{mag}$ and $J-K_{S}=$ $1.623 \pm 0.045 \mathrm{mag}$ (Skrutskie et al. 2006), its brightness and colors are normal for its spectral type. High resolution HST/ NICMOS images with good sensitivity down to separations of 0.1 arcsec show no evidence of a binary companion (Reid et al.

\footnotetext{
10 For example, Bailer-Jones \& Mundt (1999, 2001); Clarke et al. (2002a, 2002b, 2003, 2008); Gelino (2002); Gelino et al. (2002); Enoch et al. (2003); Koen (2003, 2004, 2005); Koen et al. (2004); Goldman et al. (2005); Morales-Calderón et al. (2006); Lane et al. (2007); Littlefair et al. (2008); Artigau et al. (2009); Radigan et al. (2012); Buenzli et al. (2012); Apai et al. (2013).
}

2008). Near-IR spectroscopy and $L^{\prime}$-band photometry indicate a bolometric flux of $1.10 \times 10^{-14} \mathrm{~W} \mathrm{~m}^{-2}$ (Leggett et al. 2001), with Dahn et al. (2002) finding a consistent value.

Martín et al. (1999) give $T_{\text {eff }}=1900 \mathrm{~K}$ based on optical spectroscopy, while Basri et al. (2000) find $T_{\text {eff }}=1950 \mathrm{~K}$. Dahn et al. (2002) use a bolometric luminosity derived from the measured $K_{S}$-band flux to arrive at a consistent result of $T_{\text {eff }}=1945 \pm 65 \mathrm{~K}$ (based on a radius of $0.0903 R_{\odot}$, obtained by averaging theoretical radii corresponding to the bolometric luminosity at ages of 1.0 and 5.0 Gyr). DENIS 1058 has an age of at least a few hundred Myr based on non-detections of the $6708 \AA$ lithium line down to upper limits in the equivalent width of 0.3-0.5 A (Tinney et al. 1997; Martín et al. 1997; Kirkpatrick et al. 1999). Martín et al. (1997) comment that DENIS 1058's spectral characteristics could be consistent with a very low mass $\left(0.075 M_{\odot}\right)$ star of age $3 \mathrm{Gyr}$ or a $0.065 M_{\odot}$ brown dwarf of age 800 Myr. They prefer the latter possibility based on their radial velocity measurement of $11 \pm 5 \mathrm{~km} \mathrm{~s}^{-1}$, which, like the tangential velocity of $21.1 \pm 0.4 \mathrm{~km} \mathrm{~s}^{-1}$ measured later by Dahn et al. (2002), is relatively low and therefore consistent with youth.

Of particular relevance to the measurements we report herein of DENIS 1058's photometric rotation period, Martín et al. (1997) measure its projected rotational velocity from linebroadening in their Keck HIRES spectrum, obtaining $v \sin (i)=$ $30.0 \pm 10.0 \mathrm{~km} \mathrm{~s}^{-1}$. Basri et al. (2000) perform a more sophisticated analysis of the same spectrum to find $v \sin (i)=$ $37.5 \pm 2.5 \mathrm{~km} \mathrm{~s}^{-1}$.

Denis 1058 exhibits weak $\mathrm{H} \alpha$ emission (Tinney et al. 1997; Kirkpatrick et al. 1999; Martín et al. 1999), which indicates some magnetic activity. However, the ratio of $\mathrm{H} \alpha$ to bolometric luminosity is extremely $\operatorname{low}$, with $\log \left(L_{\mathrm{H} \alpha} / L_{\mathrm{bol}}\right)=-5.67$ (Schmidt et al. 2007). Thus DENIS 1058 is not an exception to the trend of magnetic activity diminishing greatly with decreasing $T_{\text {eff }}$, which has been explored by Schmidt et al. (2007) and others. While H $\alpha$ emission in UCDs can accompany radio emission (e.g., Berger et al. 2009), we are not aware of any published radio observations of DENIS 1058.

\section{OBSERVATIONS AND DATA REDUCTION}

Our data consist of $20 \mathrm{hr}$ of photometric monitoring of DENIS 1058: first $7 \mathrm{hr}$ in the $J$-band at the $4 \mathrm{~m}$ SOAR telescope and then, three days later, $7 \mathrm{hr}$ in the Spitzer IRAC $3.6 \mu \mathrm{m}$ bandpass (hereafter [3.6]) followed immediately by $6 \mathrm{hr}$ in the IRAC $4.5 \mu \mathrm{m}$ bandpass (hereafter [4.5]). Observation specifics are presented in Table 1.

IR detectors on both Spitzer and ground-based telescopes are known to suffer from both inter-pixel and intra-pixel sensitivity variations which can create systematic errors in time-series photometry. As outlined below, our data acquisition strategies were designed to minimize such errors.

\subsection{Spitzer Data Acquisition}

For our observations with Spitzer IRAC (Fazio et al. 2004), we used the "staring mode" that has become standard for precision time-series photometry (Charbonneau et al. 2005), in which no dithering or other intentional alteration of the telescope pointing is carried out during the observing sequence. Although we were using full-frame images from IRAC, we elected to position DENIS 1058 in the upper left corner of the detector, which is the region used for subarray observations of bright targets. This was intended to give us the option 
Table 1

DENIS 1058 Photometric Monitoring Data Acquired

\begin{tabular}{|c|c|c|c|c|c|c|}
\hline \multirow[t]{2}{*}{ Wavelength } & \multicolumn{2}{|c|}{ Beginning of Observations } & \multirow{2}{*}{$\begin{array}{l}\text { Duration of } \\
\text { Monitoring }\end{array}$} & \multirow{2}{*}{$\begin{array}{c}\text { Exposure } \\
\text { Times }\end{array}$} & \multirow{2}{*}{$\begin{array}{l}\text { Images } \\
\text { Acquired }\end{array}$} & \multirow{2}{*}{$\begin{array}{l}\text { Per-image } \\
\text { rms Error }\end{array}$} \\
\hline & HMJD $^{\mathrm{a}}$ & UTC Date and Time & & & & \\
\hline$J$-band $(1.25 \mu)$ & 56000.030 & 2012 Mar 14, 00:36 & $7.01 \mathrm{hr}$ & $100 \mathrm{~s}$ & 159 & $0.30 \%$ \\
\hline IRAC [3.6] & 56003.036 & 2012 Mar 17, 00:47 & $7.33 \mathrm{hr}$ & $12 \mathrm{~s}^{\mathrm{c}}$ & 2166 & $0.59 \%$ \\
\hline IRAC [4.5] & 56003.367 & 2012 Mar 17, 08:44 & $5.85 \mathrm{hr}$ & $12 \mathrm{~s}^{\mathrm{c}}$ & 1619 & $0.76 \%$ \\
\hline
\end{tabular}

Notes.

${ }^{\mathrm{a}}$ HMJD $=$ Heliocentric Modified Julian Day.

${ }^{\mathrm{b}}$ Note that if the exposure time difference is taken into account, both IRAC bands offer precision superior to that at $J$; ground-based $J$-band measurements typically also suffer from greater systematic error.

${ }^{\mathrm{c}}$ This is the time spent integrating the whole array and is approximately equal to the sampling time. The per-pixel integration time is slightly shorter at $10.4 \mathrm{~s}$.

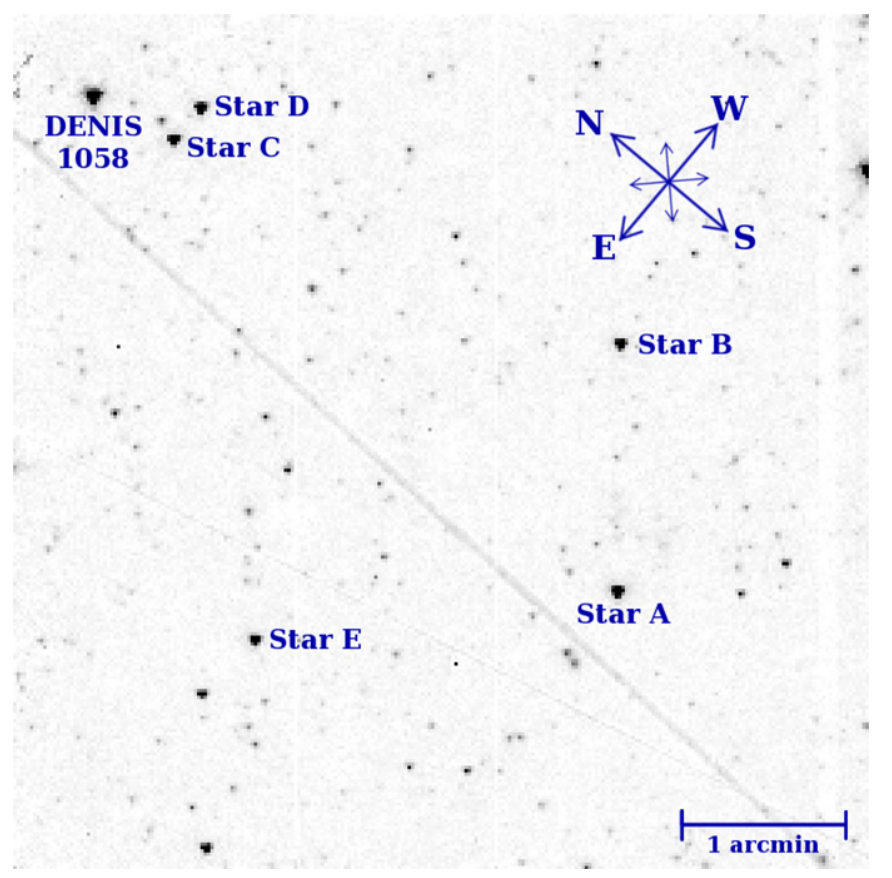

Figure 1. Stacked image made from all frames of our IRAC [3.6] data. Stars used to measure systematic errors are labeled. The streaks crossing the frame are not asteroids, because their appearance on each individual frame matches that on the full stack. They may be residual images of bright stars across which Spitzer has panned in its acquisition slew. The appearance of the starfield in the [4.5] image is similar.

(A color version of this figure is available in the online journal.)

of using the "sweet spot" calibration from the Spitzer Science Center-that is, a carefully measured sensitivity map of a specific pixel in the subarray recommended for use with precision photometry. The "sweet spot" map is still under development, and calibrations based on it have not benefited our photometric precision thus far; the methods we have actually used to remove the effects of intra-pixel sensitivity variations are described in Section 3.3. The per-image random errors in our photometry of DENIS 1058 are $0.59 \%$ and $0.76 \%$ for the IRAC [3.6] and [4.5] bands, respectively, calculated from the differences between adjacent photometric points (a difference which measures pure random noise because no known astrophysical or systematic effects are able to change the flux appreciably over the $12 \mathrm{~s}$ sampling interval). These measured values compare well with the calculated photonic shot-noise of $0.54 \%$ and $0.63 \%$ respectively: the agreement indicates that read noise, randomly varying aperture losses, and other effects make only minor contributions to the photometric error. Figure 1 shows a co-added image of all our [3.6] exposures.

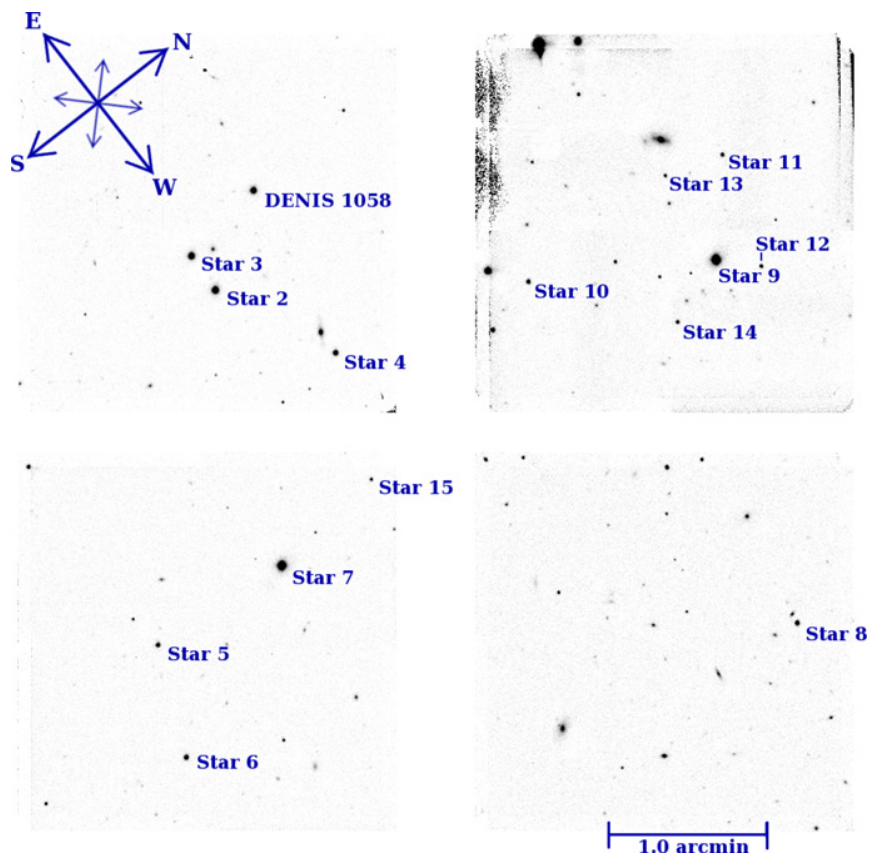

Figure 2. Stacked set of 146 of our $J$-band images of the DENIS 1058 field, with stars we considered as possible photometric references labeled. Note the separate images from the four detectors of the Spartan IR Camera, with substantial gaps that we did not attempt to fill by dithered imaging. The object immediately to the upper left of Star 4 is a galaxy; its proximity may have slightly affected the Star 4 photometry in one of the nod positions, but we believe we have successfully modeled and corrected for this effect. Note that Stars 2 and 3 here are Stars D and $\mathrm{C}$, respectively, in Figure 1. Other than this there is no overlap between the sets of comparison stars. Noisy artifacts at the vertical edges of the upper right detector are due to insensitive regions on the chip, which have no effect on any measured star.

(A color version of this figure is available in the online journal.)

\subsection{SOAR J-band Data Acquisition}

For our $J$-band observations with the SOAR telescope, we used the Spartan IR Camera (Loh et al. 2012) in its wide field configuration. The Spartan instrument uses four $2048 \times 2048$ HAWAII-2 detectors to deliver a total field of view of about $5 \times 5 \operatorname{arcmin}$ at a pixel scale of $0.068 \operatorname{arcsec}_{\text {pixel }}^{-1}$, with roughly 0.5 arcmin gaps between the detectors. Figure 2 shows a coadded image of our Spartan data. At the beginning of our observations the background level was changing rather quickly, so we elected to nod the telescope by 7 arcsec every two images, rather than take most of the images at a single pointing and interleave occasional dithered sequences. The exquisite pointing and tracking precision of the SOAR telescope allowed us to keep the two nod positions consistent with an rms pointing variation of only about one pixel $(0.07 \mathrm{arcsec})$. In a bid for 
potentially better photometric precision, we also took 14 of our 159 photometric images with the telescope defocused enough to increase the effective FWHM of our images by a bit less than a factor of two. However, as the mean FWHM of in-focus images was $\sim 10$ pixels $(0.7$ arcsec $)$, the images were already highly oversampled. The defocusing did not help; indeed, by necessitating a larger photometric aperture, it increased the background noise and rendered the photometry inferior to that obtained from in-focus images (though photometry from the defocused images remained usable).

Our images were processed by dark subtraction and flatfielding using twilight sky flats (dome flats were tried, but proved much less effective at removing the effects of dust shadows on the detector). Cosmic ray hits were numerous due to the long, 100 s exposures. We removed them using the Laplacian edge detection algorithm of van Dokkum (2001), just after the flatfielding step.

Following cosmic ray removal, we performed sky subtraction on each science image using another image taken close in time and in the opposite nod position. A scaling factor near unity was applied to each nod-subtraction image to yield a zero-mean background for the subtracted science image. The final step in our processing was to merge the images from the four detectors into a single master frame for each exposure; the stack of all in-focus merged frames is shown in Figure 2. Astrometrically, the digital gaps between the images from different detectors are only approximately correct. The per-image error on our $J$-band photometry of DENIS 1058 is $0.30 \%$ (based on the rms residuals from our final fit). This is just over twice the per-image photonic shot-noise of $0.14 \%$, which is consistent with our conclusion in Section 5 that unmodeled systematic effects remain in the $J$-band data. As described below, such effects are folded into our final uncertainties for the $J$-band analysis.

\section{SPITZER IRAC [3.5] DATA ANALYSIS}

Confirming and characterizing astrophysical variability at an amplitude comparable to that of the systematic errors requires considerable analysis, which we detail in this section. Readers interested only in the final result should skip to Section 3.4.

In Section 3.1, we describe our photometric methodology for IRAC images. In Section 3.2 we confirm the presence of astrophysical variability in DENIS 1058 by first modeling and correcting for systematic effects and then performing a Lomb-Scargle periodogram analysis, which outputs the false alarm probability (FAP), a measure of the likelihood that any apparently coherent variations are due to random noise. Comparing the FAP of DENIS 1058 to that of identically processed field stars demonstrates that DENIS 1058 is a variable. However, the periodogram is not the best tool for a detailed analysis of DENIS 1058's variations. Instead, in Section 3.3 we fit them with a Fourier model using singular value decomposition (SVD), test the robustness of the fit, and find a good parameterization for the final Markov Chain Monte Carlo (MCMC) analysis needed to calculate uncertainties, which is described in Section 3.4.

\subsection{Photometry}

Using IDL, we obtain photometry from Basic Calibrated Data images, provided by the Spitzer Science Center after processing through IRAC pipeline version 19.1.0. We obtain centroids by Gaussian fitting with the gcntrd function, setting the FWHM to 4.5 pixels to minimize the scatter in stellar positions. The fact that stellar images are actually much sharper than 4.5 pixels

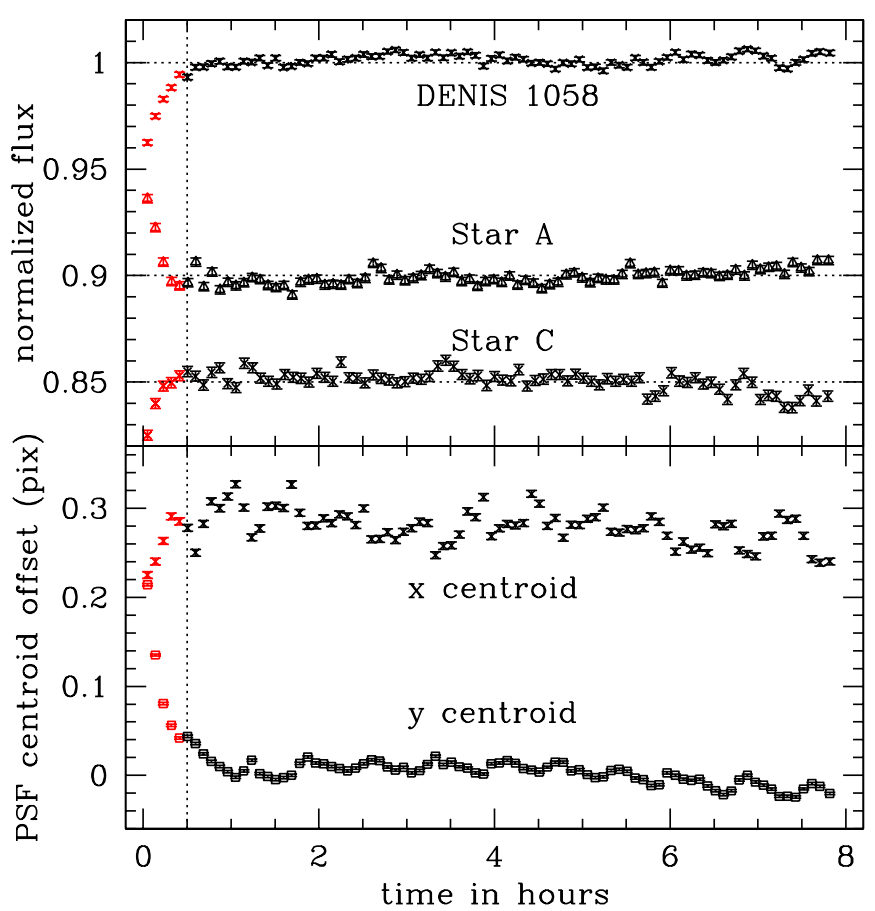

Figure 3. Upper Panel: normalized uncorrected IRAC [3.6] photometry of DENIS 1058 and two comparison stars. For clarity, the data are binned in sets of 25 points (each bin thus represents a 5 -minute time interval) and the photometry for Star A and Star C is offset by -0.1 and -0.15 , respectively, relative to DENIS 1058. Lower Panel: location of the $x$ and $y$ centroids of DENIS 1058 in the images. An offset of 0,0 corresponds to the center of pixel 23,231 on the IRAC [3.6] detector. The centroid stayed on this pixel throughout the data sequence, but its intra-pixel motion introduced systematic errors in the relative photometry due to the pixel phase effect. Previous to $t=0.5 \mathrm{hr}$ (indicated by the vertical dotted line), the spacecraft was still settling on its new pointing, and the data were not used in our final fits. Error bars are shown but in most cases are smaller than the symbols.

(A color version of this figure is available in the online journal.)

does not invalidate this choice because the gcntrd algorithm uses it only to set the size of the fitting box. We perform aperture photometry about the measured centroids with an aperture radius chosen to minimize the rms scatter of normalized photometry (2.1 pixels for DENIS 1058). Note that aperture photometry rather than point-spread function (PSF) fitting is normally used for IRAC images (e.g., Morales-Calderón et al. 2006; Todorov et al. 2012; Lewis et al. 2013); one reason for this may be IRAC's somewhat distorted "triangular" PSF.

To clip our photometric data, we first median-smooth each data vector with a sliding boxcar of width 25 points ( 6 minutes of time), and subtract this smoothed vector from the original data. All known relevant astrophysical or systematic variations have characteristic timescales longer than 6 minutes, so such signals should be absent in the subtracted vector: thus, it can be screened for outliers without danger of removing points at the extrema of variations we wish to measure. We identify outliers in the subtracted vector using the robust_sigma routine's default criterion for bad data, which (although based on the median absolute deviation) corresponds approximately to a conservative, $6 \sigma$ clip. Bad points are rejected from the original data vector, and the surviving points (both photometry and centroid positions) are binned in 10-point bins, resulting in a sampling interval of about $120 \mathrm{~s}$. Figure 3 shows the IRAC [3.6] photometry at this stage, combined with the pixel centroids.

The photometry shows the well known "pixel phase effect" in IRAC (Reach et al. 2005): the measured flux from an object 


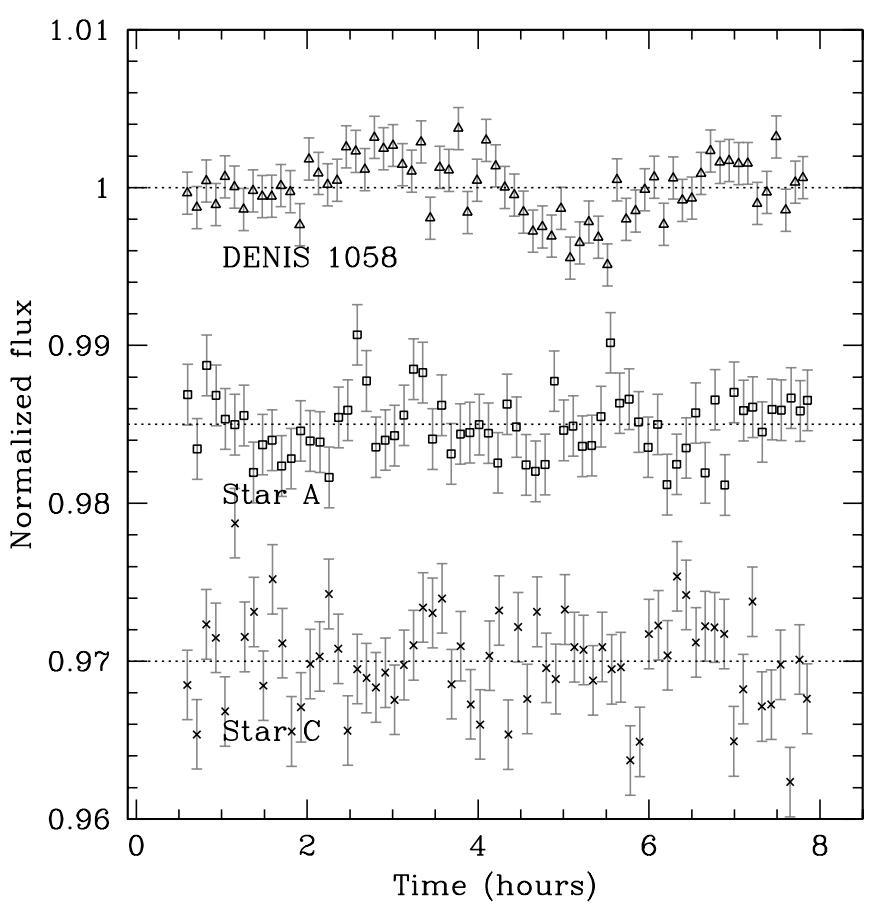

Figure 4. Normalized IRAC [3.6] photometry of DENIS 1058 and the two brightest stars in the same IRAC field, after trimming the first 30 minutes and correcting for the pixel phase effect using Equation (1). Note that this figure has a much finer vertical scale than Figure 3. For clarity, the points here are 30-point (6-minute) bins relative to the raw data, and photometry for Stars A and $\mathrm{C}$ is offset by -0.015 and -0.03 , respectively. Linear slopes and short-period oscillations present in the uncorrected data have vanished and a longer period, roughly sinusoidal variation emerges for DENIS 1058. The error bars are based on the single-point photometric errors, scaled down by $\sqrt{3} 0$ due to the binning, with an additional (minor) contribution due to the uncertainty on the centroids used in the pixel phase correction.

depends on the object's exact position within a pixel. The anomalous photometry near the beginning of the observing sequence is due to the settling of the telescope pointing after its acquisition slew, and caused us to reject the first $0.5 \mathrm{hr}$ of [3.6] data from our analysis. We note that only the pixel phase effect seems to be involved in producing this initial anomaly. It cannot be due to the asymptotic ramp phenomenon that has been reported in IRAC photometry of transiting planetary systems (see for example Knutson et al. (2009) and Todorov et al. (2012)), because the effect we observe does not have the same sign for all objects. Similarly, we have not detected any effect corresponding to the linear time trends distinct from the pixel phase effect which have previously been seen (Deming et al. 2012; Todorov et al. 2012). The absence of such effects in our data may be connected to the fact that our WoW targets are considerably fainter than the majority of transiting planetary systems targeted by Spitzer.

Following the initial settling, Spitzer's pointing shows a slow linear drift and an oscillation with a period of 0.7-1.0 hr. Both effects are reflected in the photometry, which makes correcting the pixel phase effect essential even after trimming the first $0.5 \mathrm{hr}$.

\subsection{Testing for Astrophysical Variability}

The pixel phase effect can in principle be covariant with astrophysical variability. Once we have established the presence of such variability, we address this complication by fitting for the pixel phase effect and astrophysical variations simultaneously (Section 3.3). However, without a priori knowledge of the
Table 2

Periodogram FAP Values for IRAC Data on DENIS 1058 and Field Stars

\begin{tabular}{lcccc}
\hline \hline Object & Magnitude [3.6] & Magnitude [4.5] & FAP [3.6] & FAP [4.5] \\
\hline DENIS 1058 & 11.76 & 11.76 & $2.63 \times 10^{-10}$ & $6.02 \times 10^{-1}$ \\
Star A & 12.81 & 12.84 & $4.62 \times 10^{-2}$ & $1.94 \times 10^{-1}$ \\
Star B & 13.23 & 13.18 & $6.07 \times 10^{-2}$ & $9.67 \times 10^{-1}$ \\
Star C & 13.11 & 13.12 & $2.52 \times 10^{-3}$ & $6.74 \times 10^{-1}$ \\
Star D & 13.21 & 13.14 & $5.02 \times 10^{-1}$ & $3.36 \times 10^{-1}$ \\
Star E & 13.42 & 13.50 & $4.41 \times 10^{-1}$ & $7.54 \times 10^{-1}$ \\
\hline
\end{tabular}

presence of astrophysical variability, we first implement a model only of the pixel phase effect and ratio it into our photometry.

We model the pixel phase effect as a function of both the $x$ and $y$ pixel positions (see for example Knutson et al. (2008)). Having tested functions linear in $x$ and $y$ and found them insufficient, we choose to fit a quadratic function of the form:

$$
f(x, y)=A_{0}+A_{1} x+A_{2} y+A_{3} x y+A_{4} x^{2}+A_{5} y^{2},
$$

where $f(x, y)$ models the measured flux, the $A_{i}$ are the fit coefficients, and $x$ and $y$ are sub-pixel coordinates (that is, the coordinates of the object's centroid on a given image minus the coordinates of the pixel-center nearest the object's average position over all the images). Although they move in sub-pixel coordinates, the centroids of most objects are found on the same pixel for all images, so $x$ and $y$ are normally confined to the range $(-0.5,0.5)$. We have confirmed that our fit remains effective even when this is not the case, based on results from stars in the field of DENIS 1058 and many other WoW targets. We correct our photometry by dividing by $f(x, y)$; Figure 4 shows the resulting photometry for DENIS 1058 and the two brightest field stars. DENIS 1058 exhibits roughly sinusoidal variability, while the photometry of the stars shows little evidence of coherent variations. We note that the uncertainties in the measured $x$ and $y$ centroids of DENIS 1058 (per-image values about 0.019 and 0.007 pixels, respectively) make only a minor contribution to the errors in the corrected photometry, and the same is true of the field stars.

We probe the significance of DENIS 1058's apparent variability by subjecting the corrected data to a Lomb-Scargle periodogram analysis, as implemented by Press et al. (1992). For our densely and evenly sampled data, we oversample the periodogram by a factor of 200 , but probe only up to frequencies 5 times lower than the Nyquist. As our analysis uses binned data with $120 \mathrm{~s}$ sampling, the highest frequency we probe corresponds to a 20-minute period. The periodogram of our IRAC [3.6] photometry of DENIS 1058 is the heavy line in Figure 5.

Figure 6 shows the FAP values of DENIS 1058 and 61 comparable-brightness, identically processed stars from eight different WoW fields. Although weak residual systematics prevent the FAP values from being formally accurate (i.e., a periodogram FAP of $5 \times 10^{-2}$ does not imply a $95 \%$ confidence detection of variability), the figure shows that fewer than 1 in 50 stars has an FAP value below $10^{-4}$; thus any object that does is a genuine variable with a confidence level of $\sim 98 \%$. We note that no suspected variables among the field stars have been removed from Figure 6 (only one previously known eclipsing binary), so weak astrophysical variations rather than residual systematics could be responsible for the most significant apparent detections among the stars.

With an FAP of $2.63 \times 10^{-10}$, the detection of astrophysical variability in DENIS 1058 is unambiguous. Table 2 gives the 


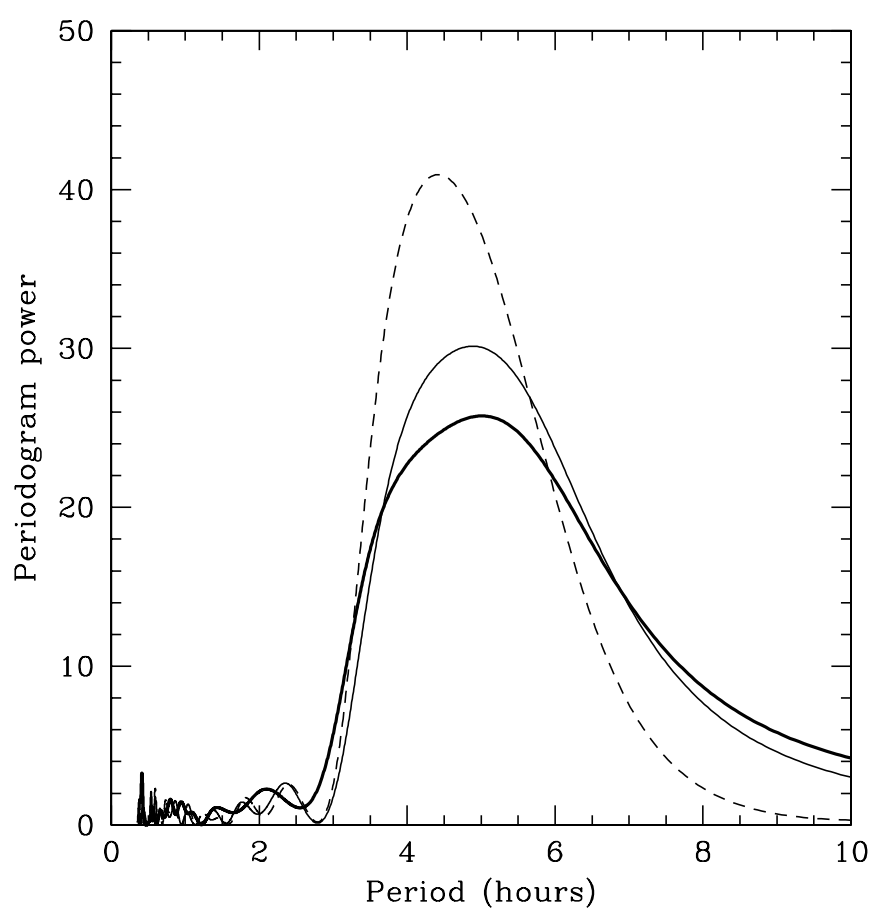

Figure 5. Periodograms of real and simulated IRAC [3.6] photometry of DENIS 1058. Heavy continuous line: periodogram of corrected real data. Dashed line: periodogram of simulated data consisting of the best-fit perfect sinusoid found in Section 3.3, with Gaussian errors added having standard deviation equal to the measured rms. Light continuous line: periodogram of simulated data after "correction" for the pixel phase effect based on the actual measured centroids of DENIS 1058. The pixel phase correction has distorted the synthetic data such that the periodogram yields an inaccurate, longer period, and it appears that the same thing has happened to the real data. The fitting methods applied in Sections 3.3 and 3.4 are not subject to this bias. Note that the width of the periodogram peaks is not trivially related to the uncertainty of the period determination.

FAP values and IRAC magnitudes for our [3.6] and [4.5] photometry of DENIS 1058 and five stars in the same IRAC field (shown as open squares in Figure 6).

Simulations we have performed, as well as actual experience with other WoW targets, indicate that pixel phase correction using Equation (1) is very unlikely to eliminate genuine astrophysical variations, although it can distort them. As shown in Figure 3, Spitzer's pointing shows both a long-term, approximately linear trend and an oscillation at a frequency of $1-1.5 \mathrm{cy}-$ cles $\mathrm{hr}^{-1}$. The extent to which the pixel phase correction can distort true astrophysical variability depends on how the timescale of the astrophysical variability compares to that of the pointing variations. The periodogram peak for DENIS 1058 is at a period of $5.02 \mathrm{hr}$, which is shorter than the observation interval but substantially longer than the pointing oscillation trend, so distortion should not be severe. However, as outlined above, we will obtain our final result from an MCMC analysis that is less prone to distortion and that allows better characterization of uncertainties than the periodogram analysis we have used simply to demonstrate that DENIS 1058 is a true variable. In fact, our MCMC analysis in Section 3.4 yields a period of $4.25_{-0.16}^{+0.26} \mathrm{hr}$, which is not consistent with the 5.02-hr periodogram peak. As illustrated in Figure 5, we have demonstrated using synthetic data that the discrepancy is indeed due to the slight distortion of the astrophysical variations that is imposed by the pixel phase correction. The $\sim 4.25 \mathrm{hr}$ value, based on the more sophisticated fits, is the correct one.

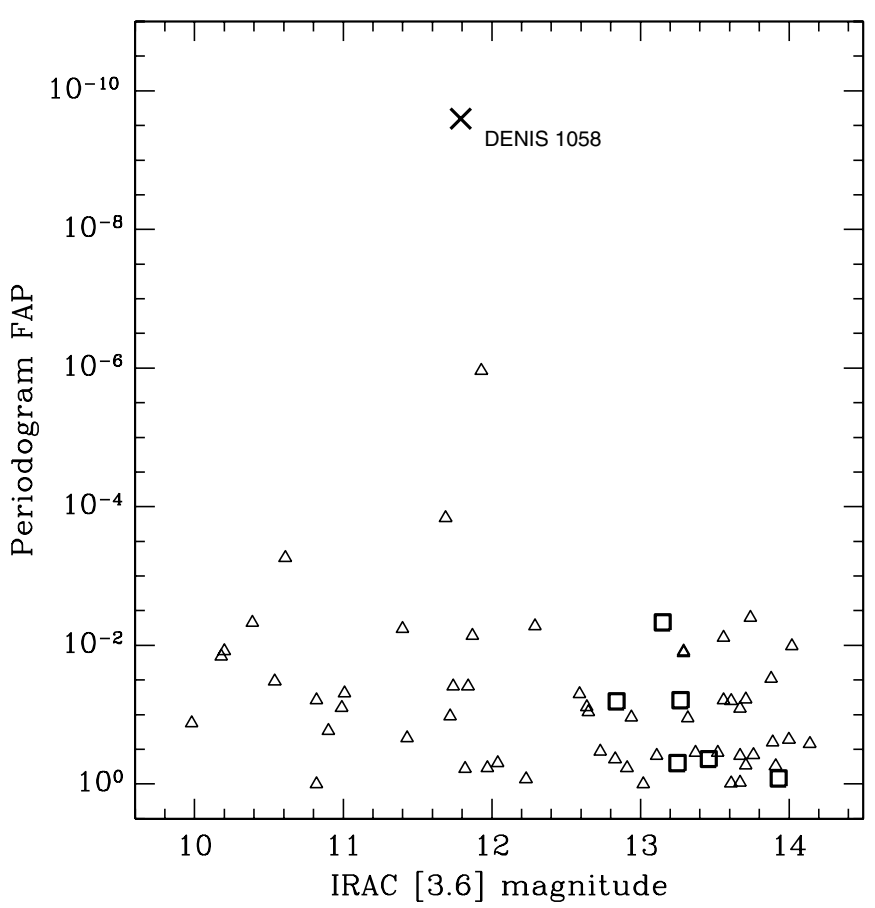

Figure 6. Periodogram FAP vs. IRAC [3.6] magnitude for field stars measured in WoW data (open symbols), and for DENIS 1058 (large "X"). DENIS 1058 shows much more significant variations than any of 61 similar-brightness stars measured in eight different WoW target fields. Stars in the same field as DENIS 1058 are shown as squares; the distribution of their FAP values is consistent with that of the stars in the other fields.

\subsection{SVD Fits to Determine Input Parameters for MCMC Analysis}

Our final analysis of DENIS 1058's variability will use an MCMC, but to determine the correct input model for such an analysis and to estimate the uncertainty on our data points, we first perform least-squares fits to the data using SVD. Our astrophysical model is a truncated Fourier series:

$$
h(t)=1+\sum_{i=1}^{n} C_{i} \cos \left(\frac{2 \pi t}{P / i}\right)+S_{i} \sin \left(\frac{2 \pi t}{P / i}\right) .
$$

Given a fixed period $P$ and photometry already corrected for the pixel phase effect, Equation (2) is linear in the parameters (the $C_{i}$ and $S_{i}$ ), so a least-squares solution could be obtained using SVD; a range of periods could be tried and the one producing the lowest residual rms identified.

However, in order to avoid distorting the astrophysical variability, we must solve simultaneously for the pixel phase parameters of Equation (1), which requires fitting the equation:

$$
g(x, y, t)=f(x, y) h(t),
$$

where $f(x, y)$ is the pixel phase function given in Equation (1). Equation (3) is nonlinear due to the multiplication of $f(x, y)$ and $h(t)$, and cannot be linearized by taking a logarithm, because both the multiplied terms are themselves the sums of independent functions.

However, since we normalize our data prior to the fit, and since the amplitudes of both systematic and astrophysical terms are small, Equation (3) can be approximated by $g(x, y, t)=$ $f(x, y)+h(t)-1$, which is linear in the parameters and thus can be solved using SVD. We take the resulting approximations for $f(x, y)$ and $h(t)$ as the starting point for an iterative solution 
of Equation (3). The first iteration begins with dividing the normalized raw photometry by the approximate value for $f(x, y)$, which produces photometry approximately corrected for the pixel phase effect. We fit this photometry using Equation (2) to yield an improved approximation of $h(t)$. We divide the raw fluxes by this, and apply Equation (1) to the resulting photometry to obtain an improved solution for $f(x, y)$ - which forms the starting point of a new iteration. This process converges rapidly even on eclipsing variables with astrophysical amplitudes greater than $20 \%$. Note that a separate iterative solution is obtained for each period in a finely sampled range, and the final output corresponds to the period that yielded the lowest residual rms. Parameter values obtained by solving the linear, approximate version of Equation (3) are always quite close to the final results: however, the residual rms is lower for the iterative solution of the full, physically self-consistent equation.

We determine the best number $n$ of Fourier terms for fitting a given data set by performing a Lomb-Scargle periodogram analysis of the residuals from the fit. We choose the lowest value of $n$ that yields an FAP for the residuals that is greater than $10^{-2}$, indicating that all measurable astrophysical variations have been successfully modeled. For DENIS 1058, fitting with only one Fourier term yields a residual FAP of 0.35 , demonstrating that a pure sinusoid is a sufficient model. The sinusoid we obtain by solving Equation (3) has a period of $4.23 \mathrm{hr}$ and a peakto-peak amplitude of $0.393 \%$. The residual $\mathrm{rms}$ from this fit is $0.186 \%$, which is identical to the value obtained by dividing the measured single-point rms (Table 1 ) by $\sqrt{1} 0$ to account for the 10 -point binning used in our analysis. This agreement indicates that all astrophysical and systematic terms have been effectively modeled. Figure 7 shows the full solution to Equation (3), together with the sinusoidal model $h(t)$ and the final residuals.

We confirm the robustness of this solution by re-solving with different initial trims, and with a cubic rather than quadratic version of the pixel-phase function $f(x, y)$. Initial trims from 10 to 40 minutes under quadratic correction, and 0.6 to 40 minutes under cubic correction, produce periods and amplitudes spanning relatively narrow ranges of $4.21-4.44 \mathrm{hr}$ and $0.388 \%-0.413 \%$, with the residual rms somewhat elevated under the least aggressive trims.

We have also experimented with photometric apertures that vary according to the value of the noise pixel parameter $\tilde{\beta}$, which measures the width of the instrumental PSF (see Lewis et al. 2013). We find that such variable apertures produce markedly poorer photometry. Similarly, including linear and quadratic terms dependent on $\tilde{\beta}$ in our fit (i.e., turning $f(x, y)$ into $f(x, y, \tilde{\beta})$ ) produces only an insignificant reduction in the standard deviation of residuals $(0.186 \%$ to $0.184 \%)$, without changing the astrophysical parameters to any substantive degree. It is not surprising that the optimal photometric analysis for DENIS 1058 would differ from that for a much brighter object such as is analyzed by Lewis et al. (2013).

\subsection{MCMC Analysis}

We subject our data to an MCMC analysis, using a nineparameter model equivalent to Equation (3). We use a constant uncertainty of $0.186 \%$ for all the data points, equal to the rms scatter from the best SVD fit. Following Ford (2005), we allow only one, randomly selected parameter to change at each link of the Markov chain, and we change it by a random amount distributed according to a Gaussian of mean zero and standard

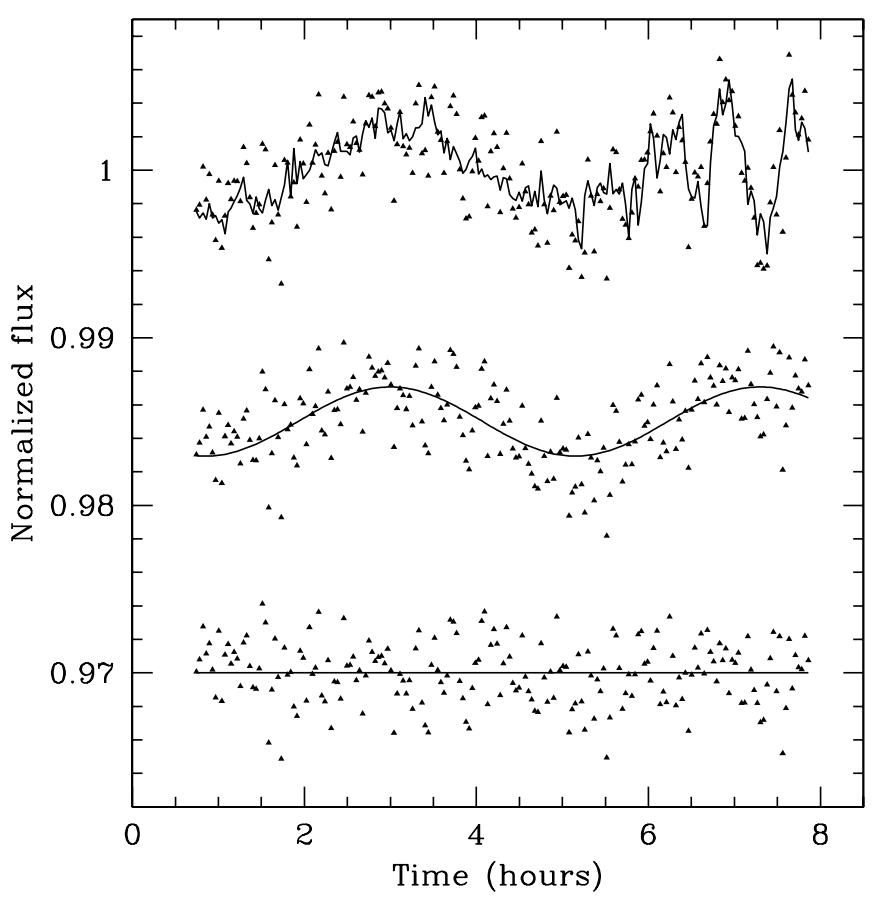

Figure 7. Top: IRAC [3.6] photometry with a simultaneous fit (Equation (3)) to both the pixel phase effect and a sinusoidal model of the astrophysical variability. Middle: photometry after correction for the pixel phase component of the fit (division by $f(x, y)$ ), with the best-fit astrophysical model $h(t)$. This sinusoidal model has a period of $4.23 \mathrm{hr}$ and a peak-to-valley amplitude of $0.393 \%$. Bottom: residuals from the full fit, which are consistent with random noise. For clarity, the corrected photometry and the residuals are offset vertically by -0.015 and -0.03 , respectively.

deviation $\beta_{\mu}$. Here $\mu$ indexes the nine fit parameters, and the $\beta_{\mu}$ values must be set appropriately before the launch of the Markov chain. We adjust them so that $20 \%-50 \%$ of the new trial values for each given parameter yield $\chi^{2}$ low enough to be accepted as a new link in the Markov chain. We have confirmed that the MCMC results are robust under different values of the $\beta_{\mu}$. They are also robust under binning schemes different from our default 10-point binning: MCMC analyses with unbinned data (12 s sampling) and 25-point binned data (5-minute sampling) produced results in agreement to well within $1 \sigma$.

We use $2 \times 10^{9}$ realizations for our final MCMC analysis. Following Ford (2005), we discard the first $10 \%$ of the Markov chain to prevent biasing the final results by not-yet-converged early solutions. We find a period of $4.25_{-0.16}^{+0.26} \mathrm{hr}$ and a peak-tovalley amplitude of $0.388 \% \pm 0.043 \%$, where $1 \sigma$ uncertainties are quoted based on the unweighted distribution of the respective parameters over all solutions accepted as links in the last $90 \%$ of the Markov chain.

\section{SPITZER IRAC [4.5] DATA ANALYSIS}

Up through the screening for astrophysical variability, the analysis of our [4.5] data proceeds almost exactly as that for the [3.6] data already described. A slightly smaller optimal photometric aperture radius (1.9 pixels) is found at [4.5], perhaps because the background noise makes a larger contribution. Because Spitzer made only a very short slew from its [3.6] pointing position, there is no pointing anomaly and no settling time at the beginning of the [4.5] data: we trim only the very first frame, which is deviant in all IRAC data sequences.

Figure 8 shows our raw binned data. Note that the time series is contiguous with that of the [3.6] data, as the WoW 


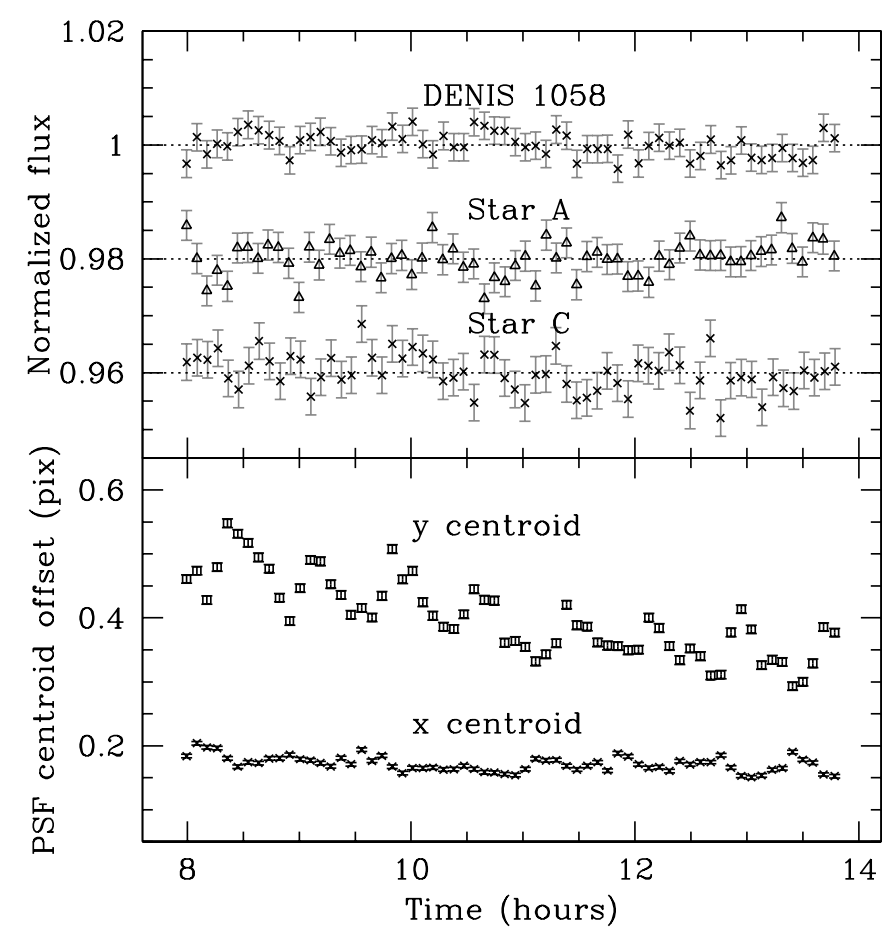

Figure 8. Upper Panel: normalized uncorrected IRAC [4.5] photometry of DENIS 1058 and two comparison stars. For clarity, the data are binned in sets of 25 points (each bin thus represents a 5 -minute time interval) and the photometry for Star A and Star C is offset by -0.02 and -0.04 , respectively, relative to DENIS 1058. Lower Panel: location of the $x$ and $y$ centroids of DENIS 1058 in the images. An offset of 0,0 corresponds to the center of pixel 23,231 on the IRAC [4.5] detector. Note that there is no initial pointing anomaly analogous to that seen in the [3.6] data.

observations of each target are sequential. The pixel phase effect is weaker in [4.5] versus [3.6], but correction is still warranted. Figure 9 shows the data after correction using Equation (1).

The FAP values of our [4.5] data are given in Table 2. With two of five measured field stars showing lower FAP, there is no evidence that DENIS 1058 exhibits significant variability at [4.5]. We note that this conclusion is unaffected by the fact that thanks to weaker systematics and a shorter data sequence, FAP values tend to be higher for all objects at [4.5] versus [3.6]. As we will demonstrate below, this non-detection is not due to lower sensitivity at the longer passband: variability with the same amplitude as at [3.6] would easily be detected in our [4.5] data.

We emphasize that DENIS 1058 is variable at [3.6] beyond reasonable doubt, as previously demonstrated by Figure 6. We note that Morales-Calderón et al. (2006) also found two L dwarfs that showed possible variations in one Spitzer IRAC band but not in another (the bands were [4.5] and [8.0], respectively). Because of the lack of confirmation at [8.0], they refrained from claiming their [4.5] detection as true astrophysical variability. While such caution was warranted then, our own [3.6] detection is confirmed by a systematic analysis of field stars that was beyond the scope of previous work. Since our result shows that the variability amplitude of an L dwarf can be very different from one band to another, it may suggest that the variations reported by Morales-Calderón et al. (2006) at [4.5] had a genuine astrophysical origin.

Our periodogram analysis suggests that our [4.5] photometry of DENIS 1058 is consistent with zero variability, and Figures 8 and 9 support this. An MCMC analysis of the same form as we used for the [3.6] data would thus find sinusoidal amplitudes consistent with zero and therefore fail to converge on meaningful

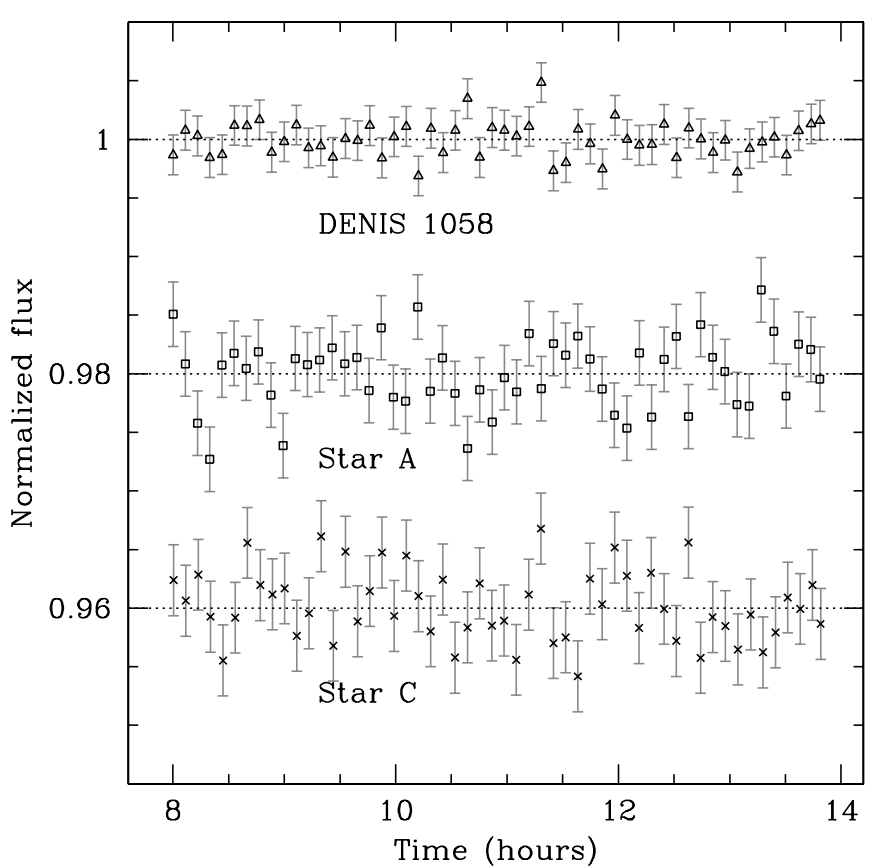

Figure 9. Binned, normalized IRAC [4.5] photometry of DENIS 1058 and the two brightest stars in the same IRAC field, after correction for the "pixel phase" effect based on Equation (1). For clarity, the points here are 30-point (6-minute) bins relative to the raw data, and photometry for Stars A and $\mathrm{C}$ is offset by -0.015 and -0.03 , respectively. Weak pixel-phase artifacts visible in Figure 8 are well-corrected, but in contrast to the [3.6] results, DENIS 1058 shows no evidence for variability in [4.5].

values for the period and phase. To avoid this, we perform an MCMC analysis with the period and phase fixed to the final values from [3.6] analysis. Thus our [4.5] MCMC analysis has seven rather than nine parameters: the six $A_{i}$ from Equation (1) plus only the amplitude of the sinusoid.

This analysis yields a peak-to-valley amplitude of $0.090 \% \pm$ $0.056 \%$, which corresponds to a [4.5]/[3.6] amplitude ratio of $0.23 \pm 0.15$. While the positive amplitude ratio suggests that DENIS 1058 exhibits weak [4.5] variability positively correlated with that at [3.6], we note that the data are consistent with zero and even negative amplitudes (corresponding to anti-correlated variations). By contrast, [4.5] variations with amplitude equal to those at [3.6] are excluded at the $5 \sigma$ level. Figure 10 combines the data and best-fit sinusoids for both [3.6] and [4.5], illustrating the striking difference in DENIS 1058's photometric behavior in the two bands.

\section{SOAR $J$-BAND ANALYSIS}

We begin our $J$-band analysis of DENIS 1058 by identifying 14 field stars bright enough to be potentially useful for relative photometry. They are shown in Figure 2 and listed in Table 3.

We proceed to construct relative photometry of DENIS 1058 by ratioing its flux on each image to the sum over measured fluxes of all 14 reference stars on the same image. To screen for variability and explore the systematic effects present in our data, we also construct relative photometry of the reference stars by ratioing the flux of each to the summed flux of all the others:

$$
R_{j i}=\frac{F_{j i}}{\sum_{k=2, k \neq j}^{m} F_{k i}} .
$$

Here, $i$ indexes images while $j$ and $k$ index objects measured on each image, with $j=1$ for DENIS 1058 itself. The $F_{j i}$ are 
Table 3

Objects Measured in SOAR $J$-band Images

\begin{tabular}{|c|c|c|c|c|c|c|c|}
\hline \multirow{2}{*}{$\begin{array}{l}\text { Designation } \\
\text { (This Work) }\end{array}$} & \multirow{2}{*}{$\begin{array}{c}\text { Catalog } \\
\text { Designation }^{\mathrm{a}}\end{array}$} & \multicolumn{2}{|c|}{ J2000.0 Coordinates } & \multirow{2}{*}{$\begin{array}{c}\text { 2MASS } J \\
\text { (mag) }\end{array}$} & \multirow{2}{*}{$\begin{array}{c}\text { 2MASS } J-K_{S} \\
(\mathrm{mag})\end{array}$} & \multirow[t]{2}{*}{$F^{\mathrm{b}}$} & \multirow{2}{*}{$\begin{array}{c}\text { SOAR } \\
\text { Relative rms }\end{array}$} \\
\hline & & R.A. & Decl. & & & & \\
\hline DENIS 1058 & 2MASS J10584787-1548172 & $10: 58: 47.87$ & $-15: 48: 17.2$ & $14.16 \pm 0.04$ & $1.62 \pm 0.05$ & 20.11 & $1.07 \%$ \\
\hline Star 2 & 2MASS J10584625-1548513 & $10: 58: 46.26$ & $-15: 48: 51.4$ & $13.59 \pm 0.03$ & $0.43 \pm 0.05$ & 14.43 & $0.72 \%$ \\
\hline Star 3 & 2MASS J10584730-1548500 & $10: 58: 47.30$ & $-15: 48: 50.0$ & $13.88 \pm 0.03$ & $0.76 \pm 0.05$ & 15.23 & $0.54 \%$ \\
\hline Star 4 & 2MASS J10584309-1548310 & $10: 58: 43.09$ & $-15: 48: 31.1$ & $14.81 \pm 0.04$ & $0.45 \pm 0.11$ & 15.63 & $1.18 \%$ \\
\hline Star 5 & 2MASS J10583944-1550392 & $10: 58: 39.44$ & $-15: 50: 39.3$ & $16.06 \pm 0.09$ & $0.60 \pm 0.25$ & 17.30 & $1.37 \%$ \\
\hline Star 6 & 2MASS J10583674-1550562 & $10: 58: 36.74$ & $-15: 50: 56.3$ & $15.95 \pm 0.07$ & $0.85 \pm 0.18$ & 17.01 & $0.89 \%$ \\
\hline Star 7 & 2MASS J10583905-1549451 & $10: 58: 39.05$ & $-15: 49: 45.2$ & $12.87 \pm 0.02$ & $0.80 \pm 0.04$ & 14.46 & $0.85 \%$ \\
\hline Star 8 & 2MASS J10582955-1547299 & $10: 58: 29.55$ & $-15: 47: 29.9$ & $16.23 \pm 0.10$ & $\sim 0.77^{\mathrm{d}}$ & 16.77 & $1.31 \%$ \\
\hline Star 9 & 2MASS J10583882-1546204 & $10: 58: 38.83$ & $-15: 46: 20.5$ & $12.56 \pm 0.02$ & $0.41 \pm 0.03$ & 13.46 & $1.03 \%$ \\
\hline Star 10 & 2MASS J10584149-1547206 & $10: 58: 41.50$ & $-15: 47: 20.7$ & $16.16 \pm 0.10$ & $0.64 \pm 0.25$ & 17.23 & $1.31 \%$ \\
\hline Star 11 & GSC 2.3 S5IT005181 & $10: 58: 40.78$ & $-15: 45: 53.7$ & $\ldots$ & $\cdots$ & 18.56 & $2.14 \%$ \\
\hline Star 12 & GSC 2.3 S5IT005141 & $10: 58: 37.94$ & $-15: 46: 08.8$ & $\cdots$ & $\cdots$ & 18.50 & $2.80 \%$ \\
\hline Star 13 & GSC 2.3 S5IT005115 & $10: 58: 41.32$ & $-15: 46: 15.3$ & $\cdots$ & $\cdots$ & 19.64 & $4.14 \%$ \\
\hline Star 14 & GSC 2.3 S5IT005041 & $10: 58: 38.22$ & $-15: 46: 46.5$ & $\cdots$ & $\cdots$ & 18.23 & $3.87 \%$ \\
\hline Star 15 & GSC 2.3 S5IT004646 & $10: 58: 39.33$ & $-15: 48: 59.6$ & $\cdots$ & $\cdots$ & 20.09 & $3.53 \%$ \\
\hline
\end{tabular}

Notes.

a DENIS 1058 and Stars 2-10 were found in the 2MASS catalog; the rest of the stars had no 2MASS detections but were found in the GSC 2.3 catalog.

${ }^{\mathrm{b}}$ Red photographic magnitudes from the POSS. Uncertainties are typically $0.4-0.5$ mag.

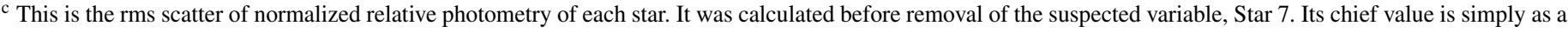
rough relative metric for the random scatter of each object.

d There is a photometry error flag on the 2MASS $K$-band magnitude of this object. Its color is thus uncertain.

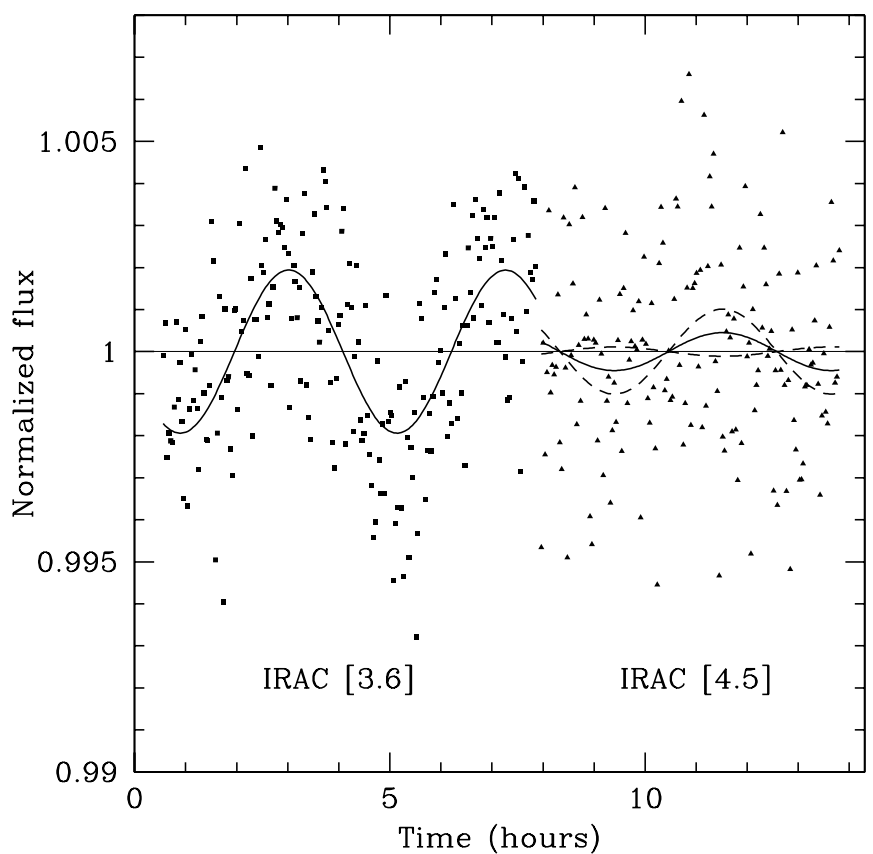

Figure 10. IRAC [3.6] and [4.5] data after removal of the pixel phase systematics based on simultaneous fits to the pixel phase effect and a sinusoid. The best fit sinusoids for both bands are shown as solid lines, where the [4.5] curve has been constrained to have the same period and phase as that at [3.6]. The dashed curves give the minimum and maximum amplitudes permitted for the [4.5] data at the $2 \sigma$ level. The former amplitude is negative and corresponds to extremely low-amplitude variations anti-correlated with those observed at [3.6], but demonstrates that the data are also consistent with zero variability at [4.5]. The data suggest weak positively correlated variability, but zero or anti-correlated variability cannot be ruled out.

instrumental fluxes while the $R_{j i}$ are the relative photometry. We optimize our photometric apertures to minimize the rms scatter of the $R_{j i}$ for field stars with similar brightness to DENIS 1058. This results in an aperture of radius 13 pixels $(0.88$ arcsec $)$ for our in-focus images and 17 pixels $(1.16$ arcsec $)$ for the defocused

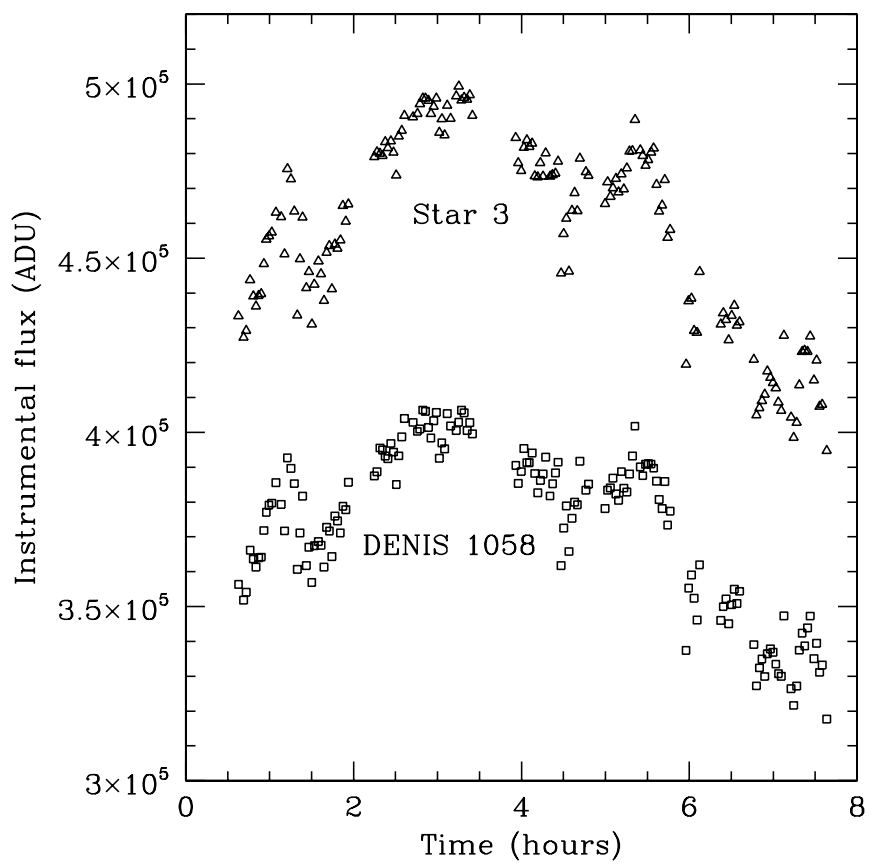

Figure 11. Instrumental $J$-band photometry of DENIS 1058 and a similarbrightness field star from our SOAR images. The $\sim 20 \%$ range of variation seen here is mainly due to variable aperture losses, which cancel out when relative photometry is constructed by ratioing the flux of DENIS 1058 to the summed fluxes of a set of non-variable field stars, including the star shown here.

images mentioned in Section 2.2. For sky subtraction we use an annulus of inner radius 55 pixels and width 10 pixels around each star.

Figure 11 shows our raw photometry of DENIS 1058 and Star $3\left(F_{1 i}\right.$ and $\left.F_{3 i}\right)$ as a function of time. The variations are caused predominately by changing aperture losses due to seeing, telescope flexure, and focus adjustments. Airmass plays a secondary role, and both effects cancel out when the data are ratioed. 


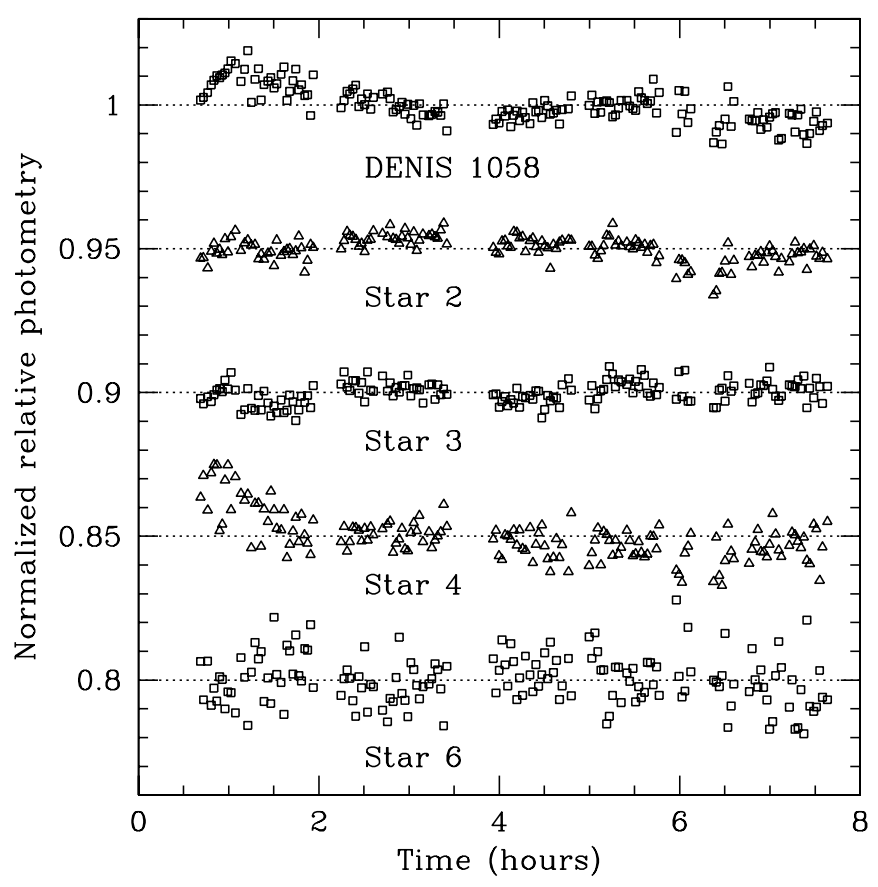

Figure 12. Normalized relative photometry of DENIS 1058 and several bright field stars in the $J$-band, after correction for the photometric offset between the two nod positions. In the plot, we have offset the data for each star by -0.05 relative to the previous one, for clarity. DENIS 1058 exhibits two welldefined local extrema (at $\sim 3.8$ and $\sim 5.7 \mathrm{hr}$ ) that are not at the endpoints of the time series. This property is consistent through many different ways of fitting the data, and is not shared by the field stars. Data in the range 5.9-6.6 hr were taken with the telescope purposely defocused; the larger photometric aperture required for these data increased the noise.

Our initial relative photometry shows deviant behavior for Star 7 and Star 9, the two brightest objects in the field. Further investigation shows that Star 9 occasionally saturates, while Star 7 appears to be a variable. We reject both stars as photometric references.

With these stars rejected, the largest remaining systematic variations take the form of a clear bimodality in the relative fluxes from images taken in one nod position versus the other. This is not unexpected due to the likely existence of differing flatfield residuals at the two nod positions. While the amount of the offset differs from one star to another, it appears constant in time for each star and is therefore easy to correct. Because we nodded the telescope every $\sim 240 \mathrm{~s}$ during the data acquisition, the correction has no risk of distorting any but the highest frequency astrophysical variations (e.g., asteroseismic pulsations) which, if present, would have photometric amplitudes too small to be relevant here. Figure 12 shows relative photometry of DENIS 1058 and the four brightest non-variable field stars after correction for this nod-offset effect.

DENIS 1058 already appears more variable than the other stars, with the suggestion of a 4-5 hr sinusoidal variation consistent with the IRAC [3.6] results (though at a larger amplitude). However, as three days elapsed between the SOAR and Spitzer observations, and the [3.6] period is not sufficiently accurate to preserve phase information over this time interval, we analyze the $J$-band data independently of the [3.6] results.

Figure 12 indicates some residual systematics in the photometry of the field stars, as well as an apparent linear fading trend superimposed on the approximately sinusoidal variations of DENIS 1058. We have explored the origin of these systematics by multilinear SVD fits to the normalized relative photometry of each object. Beyond the systematic offset between nod positions discussed above, the systematic errors in our photometry show no clear correlation with airmass, pixel position, or image sharpness. Linear fits to relative photometry as a function of either airmass or time yield some reduction in the residual standard deviation. However, they do not correct all systematic errors, and the linear time trend consistently produces a greater improvement than the airmass or pixel-position fits. A period near $4 \mathrm{hr}$ and an amplitude near $1 \%$ are robustly found for DENIS 1058 under correction by either a linear fit to airmass or a linear time trend; this fit is also robust whether relative photometry is constructed by ratioing DENIS 1058 to the sum of all non-variable reference stars or only to the flux of any single star among the three brightest (Stars 2-4). While the systematic variations in the field stars can be fit by sinusoids, no such sinusoidal fit approaches the consistency of the fit to DENIS 1058 under different photometric ratios and selections of systematic parameters.

We emphasize that there is no evidence that any of the relative photometry has a physically reasonable dependence on airmass. In particular, despite DENIS 1058 's very different $J-K_{S}$ color relative to all the reference stars, the sign of the airmass term is not consistent in fits to relative photometry constructed by ratioing the $\mathrm{L}$ dwarf to different individual reference stars. This is consistent with the fact that the bandpass of the MKO $J$ filter used in the Spartan IR Camera does not include wavelengths affected by strong telluric water-vapor absorption. The Earth's atmosphere has, in fact, almost a uniform opacity across this band, which implies that objects of very different colors will nonetheless experience identical airmass effects, consistent with what we observe.

We choose to model the systematics of our $J$-band photometry using two parameters: the nod offset correction plus a linear time trend. This is by far the best two-parameter model, and while it does not correct all the systematic effects seen in the photometry of the field stars, we feel that a more aggressive choice would be too likely to distort the fit to DENIS 1058's true astrophysical variability.

Since our model cannot remove all the systematic effects, we created an iterative process to reduce them as much as possible, obtaining improved relative photometry to input to our fits for both systematic and astrophysical variations. The objective of this process was to prevent individual deviant points and systematic effects specific to a particular star from affecting the relative photometry of the others. The process is described in detail in the Appendix. We emphasize that it has no ability to remove either systematic errors or astrophysical variations specific to a given object from that object's final photometry. Its sole purpose is to reduce the effect that deviant photometry of the reference objects has on the relative photometry of any given star. Improvements to the photometry were subtle, but the occurrence rates of photometric outliers and the standard deviations of fit residuals were reduced. As a final step, we fit a cubic polynomial in time to each object, and removed all $2.5 \sigma$ outliers from this fit. This fit was for purposes of trimming only and was not a correction applied to the data. The maximum number of points clipped was 4 out of 159 .

We fit a sinusoid to our corrected $J$-band photometry of DENIS 1058 and each of the four brightest field stars, using the same algorithm as for our IRAC [3.6] data to fit simultaneously for the systematic error terms. Consistent with our initial results on the robustness of sinusoidal fits to DENIS 1058's $J$-band photometry, we find that although best-fit sinusoids do of course 


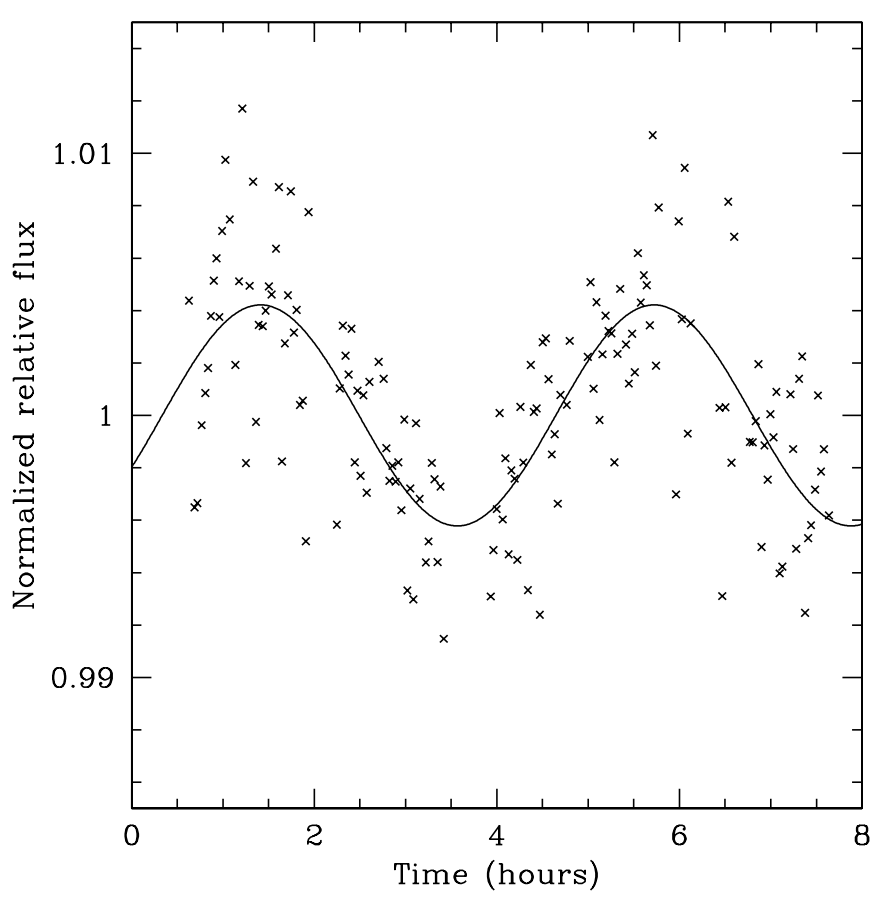

Figure 13. Final normalized relative photometry of DENIS 1058 in the $J$-band, corrected by the nod position offset and a linear time trend, and fit with the best-fit sinusoid, having a period of $4.31 \mathrm{hr}$ and a peak-to-valley amplitude of $0.843 \%$

formally exist for all the field stars, the sinusoidal component of the fit produces the most significant reduction in the residual standard deviation for DENIS 1058. The best-fit period for DENIS 1058 in the $J$-band is $4.31 \pm 0.31 \mathrm{hr}$, and the peakto-valley amplitude is $0.843 \% \pm 0.098 \%$. Figure 13 shows this final fit.

Due to the presence of systematic effects for which we had no physical model, quantifying the uncertainties we have quoted above required a different approach from the MCMC analysis we applied to the IRAC data. Instead, we used the four brightest reference stars to obtain a sampling of the typical systematic errors, imposed these errors on the photometry of DENIS 1058, and measured the resulting scatter in the sinusoidal fit parameters. To do this, we modeled the normalized photometry of each star using a cubic function of time and then multiplied the relative photometry of DENIS 1058 by this function. In this way we created four different realizations of $J$-band photometry for DENIS 1058, each with the systematic errors of a different reference star imposed. We fitted the resulting distorted photometry using the same method applied to the original data. Thus we obtained five different values for each parameter of the sinusoid: one from the original photometry and one from each systematically altered version. The uncertainties quoted above are the standard errors from these five values.

Because of the systematics in our SOAR data and the low amplitude of DENIS 1058's variability, if we did not have the IRAC [3.6] data as well, we might report only a tentative detection of periodic $J$-band variability despite the multiple lines of evidence in the $J$ band data that point to its reality. Many such tentative detections of L dwarf variability have been made, which are probably real based on statistical arguments (e.g., Gelino et al. 2002; Koen 2003, 2004, and 2005). However, given our IRAC [3.6] detection at a period matching the independently derived $J$-band period within $1 \sigma$, the reality of DENIS 1058 's $J$-band periodic variability is confirmed.
The amplitude of DENIS 1058's variability is higher in the $J$-band than in IRAC [3.6] by a factor of $2.17 \pm 0.35$. The fact that the amplitude is higher in the $J$-band relative to longer wavelengths is consistent with theoretical models of cloudinduced variability, and also matches observations of other variable brown dwarfs (Radigan et al. 2012; Buenzli et al. 2012; Apai et al. 2013).

\section{ASTROPHYSICAL IMPLICATIONS}

DENIS 1058 varies in both the IRAC [3.6] and $J$-bands with a consistent period but with substantially different amplitudes. No significant variability is detected in IRAC [4.5], and any variability in this band that is in phase with the [3.6] light curve must (with $95 \%$ confidence) have no more than about half the [3.6] amplitude. We now consider the origins of DENIS 1058's variability.

Periodic variability in stars is usually due to one of three types of phenomena: close binaries and planetary companions (either eclipsing or ellipsoidal variables), stellar pulsation, or rotation combined with magnetic star spots. L dwarfs, being cool enough to form condensate clouds, can also exhibit periodic variability due to rotation combined with inhomogeneous cloud cover.

The significant differences in amplitude at different wavelengths in our data suggest that the observed variability cannot be due to global changes in DENIS 1058, such as those due to tidal effects from a close binary or to pulsation. Marley et al. (1990) also find that the longest possible pulsational periods for brown dwarfs are at least a factor of two shorter than the period we have observed. This leaves some type of rotational variability, induced either by magnetic phenomena or inhomogeneous clouds, as the preferred model. In either case the variability has provided us with a rotation period. Thus, before we consider the likely origin of the variability in more detail, we first constrain the radius and age of DENIS 1058 based on the Basri et al. (2000) measurement of its projected rotational velocity.

\subsection{Radius and Age}

Basri et al. (2000) measure the projected rotational velocity of DENIS 1058 to be $v \sin (i)=37.5 \pm 2.5 \mathrm{~km} \mathrm{~s}^{-1}$ based on line broadening in their Keck/HIRES spectrum. Given rotation period $P$ and equatorial rotation velocity $v$, an object's radius is $R=P v /(2 \pi)$. Since $v \sin (i)$ constitutes a lower limit on the true rotational velocity, we can use it to get a lower limit on the radius of DENIS 1058. Using our IRAC [3.6] period of $4.25_{-0.16}^{+0.26} \mathrm{hr}$, we find that $R=0.131_{-0.010}^{+0.012} R_{\odot}$, which yields a $2 \sigma$ lower limit of $0.111 R_{\odot}$.

This lower limit radius permits us to set upper limits on the age and mass of DENIS 1058, using the fact that brown dwarfs contract over time and more massive ones have smaller radii at a given temperature. The only additional input we

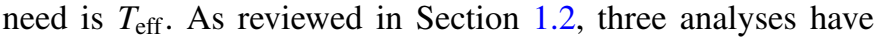
consistently found $T_{\text {eff }} \sim 1950 \mathrm{~K}$. Only Dahn et al. (2002) quote an uncertainty, finding $T_{\text {eff }}=1945 \pm 65 \mathrm{~K}$. Using the evolutionary models of Saumon \& Marley (2008) for objects of solar metallicity and $f_{\text {sed }}=2$ (generally a good fit for L dwarfs; see Stephens et al. 2009), we find that the largest-mass (and oldest) model consistent with our radius limit has age $320 \mathrm{Myr}$, mass $0.055 M_{\odot}$, luminosity $1.90 \times 10^{-4} L_{\odot}, \log (g)=5.09$, and $T_{\text {eff }}=2030 \mathrm{~K}$.

This model is, however, inconsistent with other data. First, the luminosity is much too high. The results of Leggett et al. (2001) and Dahn et al. (2002) allow us to calculate the bolometric 
luminosity of DENIS 1058 at $(1.03 \pm 0.07) \times 10^{-4} L_{\odot}$, where we have set the uncertainty on the bolometric correction to $5 \%$. This is inconsistent with the 320 Myr model by $12 \sigma$ (neglecting uncertainties in the model luminosity). Secondly, a $320 \mathrm{Myr}$ age is probably inconsistent with the lithium non-detections reported by Tinney et al. (1997), Martín et al. (1997), and Kirkpatrick et al. (1999). We suggest two possible resolutions to the discrepancy, in the form of two parameters that, when all available uncertainties are considered, show only a $\sim 3 \sigma$ disagreement.

Firstly, we consider $T_{\text {eff }}$. Adopting our lower-limit radius, we can use the measured luminosity and the equation $T_{\text {eff }}=$ $\left(L / 4 \pi R^{2} \sigma\right)^{1 / 4}$ to find $T_{\text {eff }}=1750 \pm 30 \mathrm{~K}$, which differs from the Dahn et al. (2002) result by only $2.7 \sigma$. We note also that Dahn et al. (2002) adopted a radius of $0.0903 R_{\odot}$ in their calculation, and that a cooler $T_{\text {eff }}$ will apply if the true radius is larger. Martín et al. (1999) and Basri et al. (2000) report $T_{\text {eff }}=1900 \mathrm{~K}$ and $1950 \mathrm{~K}$, respectively, but the uncertainties on these values may be large enough not to be inconsistent with $T_{\text {eff }}=1750 \pm 30 \mathrm{~K}$. More problematically, however, an object with $T_{\text {eff }}=1750 \pm 30 \mathrm{~K}$ and $R=0.111 R_{\odot}$ would have age and mass well below $320 \mathrm{Myr}$ and $0.055 M_{\odot}$, exacerbating the inconsistency with lithium non-detections.

Secondly, therefore, we consider the rotation speed. Tinney et al. (1997) comment that a model with mass $0.065 M_{\odot}$ and age 800 Myr would be consistent with their spectral data. This statement still holds based on the Saumon \& Marley (2008) models, which indicate that such an object would have $T_{\text {eff }}$ and luminosity consistent with measurements. Its radius would be $0.100 R_{\odot}$, which we can combine with our period to find a rotational velocity of $28.6_{-1.6}^{+1.1} \mathrm{~km} \mathrm{~s}^{-1}$. This disagrees with the Basri et al. (2000) value by only $3.3 \sigma$, and we note that this is without including in the error propagation any estimate for the uncertainty on the theoretical radius.

Regardless of which (if either) of these possible resolutions for the discrepancy is to be preferred, our large radius estimate for DENIS 1058 demonstrates that our viewing geometry must be approximately equator-on. Changing the assumed inclination from $90^{\circ}$ to $45^{\circ}$, for example, yields $R=0.186 \pm 0.011 R_{\odot}$, which could be reconciled with the measured luminosity only by adopting a $T_{\text {eff }}$ of less than $1450 \mathrm{~K}$. Such a value would be inconsistent with the observed spectral type and would also imply a very young, low-mass object that should show prominent lithium absorption. Thus, while the discrepancy described above prevents us from placing a formally precise limit on the inclination of DENIS 1058, a value of at least $45^{\circ}$ is strongly implied.

\subsection{Photospheric Spots and Clouds}

In this section we consider inhomogeneous clouds and/or magnetic starspots as possible causes of the variability we observe in DENIS 1058. Cool starspots are produced when locally strong magnetic fields inhibit convective heat transport in the stellar atmosphere. As we discuss below in Section 6.3, they may not be able to form in L dwarfs, but for purposes of the present analysis we will grant them to be at least a possibility. Warm spots could arise from the deposition of magnetic energy in the photosphere (producing continuum emission) or the chromosphere (producing line emission). There are no published observations of the former (that is, persistent photospheric warm spots of probable magnetic origin); nevertheless we will consider the possibility briefly in the current section. Variability due to magnetic line emission will be considered in Section 6.4.
If DENIS 1058's variability is due to photospheric spots with a large temperature differential, we would expect them to exhibit high surface brightness contrast across a wide range in wavelengths. This is inconsistent with the large differences in observed variability amplitude between [4.5], [3.6], and the $J$-band. For example, although the [3.6] and $J$-band variability of DENIS 1058 could be explained by a photospheric warm spot with a $T=2880 \pm 210 \mathrm{~K}$ blackbody spectrum, ${ }^{11}$ this scenario overpredicts the variability amplitude at [4.5] by more than $6 \sigma$. More sophisticated modeling described below reaches the same conclusion: neither cold nor hot spots, regardless of the temperature differential, can explain the observed variability in the absence of inhomogeneous clouds.

Following similar analyses performed on T dwarfs by Artigau et al. (2009), Radigan et al. (2012), and Apai et al. (2013), we construct a two-phase model of DENIS 1058: a primary phase modeling the expected global overcast, and a secondary phase with different temperature and/or cloud parameters. For both phases we use different model spectra from Saumon \& Marley (2008). A spectral fit to establish the temperature and cloud properties appropriate for the primary phase is beyond the scope of this work. Instead, we rely on existing analyses that have consistently found $T_{\text {eff }} \sim 1950 \mathrm{~K}$ for DENIS 1058 , remembering also that its $J-K_{S}$ color gives no indication of unusual atmospheric properties. Thus, for the primary phase we use models with $T_{\text {eff }}=1950 \mathrm{~K}, \log (g)=5.0$, and cloud parameters corresponding to moderately thick clouds $\left(f_{\text {sed }}=1\right.$, 2 , or 3), consistent with those that, e.g., Stephens et al. (2009) have found to match $\mathrm{L}$ dwarfs with spectral types similar to DENIS 1058. We note that most of the models considered in Section 6.1 had $\log (g)$ fairly close to 5.0. If DENIS 1058 matches the young model with $T_{\text {eff }}=1750 \mathrm{~K}$, the spectral models we consider in the current section will be somewhat incorrect, but the basic conclusions should still apply.

The Saumon \& Marley (2008) theoretical spectra that we consider for modeling the secondary phase have $\log (g)=5.0$, with $T_{\text {eff }}$ values ranging from 1500 to $2300 \mathrm{~K}$ in intervals of $100 \mathrm{~K}$, and five different values for the cloudiness parameter $f_{\text {sed }}: 1,2,3,4$, and $\infty$. The latter quantity parameterizes the extent to which sedimentation, or rain, occurs for the clouds: thus the models with $f_{\text {sed }}=1$ have the thickest clouds (fewest cloud particles removed by sedimentation) while $f_{\text {sed }}=\infty$ corresponds to a completely cloudless case. We have interpolated logarithmically in $T_{\text {eff }}$ to obtain models with a spacing of $10 \mathrm{~K}$.

We seek to match three observables: our IRAC [3.6] amplitude, our [4.5]/[3.6] amplitude ratio, and our $J /[3.6]$ amplitude ratio. For a given primary phase/secondary phase pair, we integrate the spectral models over the bandpasses of the IRAC [3.6], IRAC [4.5], and MKO $J$ filters used in our observations to get fluxes in each filter for each model. We then assume that one side of DENIS 1058 is completely covered by the primary phase, while the other side has secondary-phase regions extending over a fractional area $\epsilon$. We solve for $\epsilon$ based on the [3.6] amplitude. Let $p$ be the primary-phase model flux integrated over the IRAC [3.6] band, and $s$ be the secondary-phase model integrated over the same band. Then the amplitude of variation is:

$$
A_{[3.6]}=\frac{(1-\epsilon) p+\epsilon s-p}{p} .
$$

\footnotetext{
11 Magnitudes were converted to fluxes for use in this calculation (and that in Section 6.4 below) based on information from the IRAC Instrument Handbook (http://irsa.ipac.caltech.edu/data/SPITZER/docs/irac/iracinstrumenthandbook) and from Cohen et al. (2003).
} 


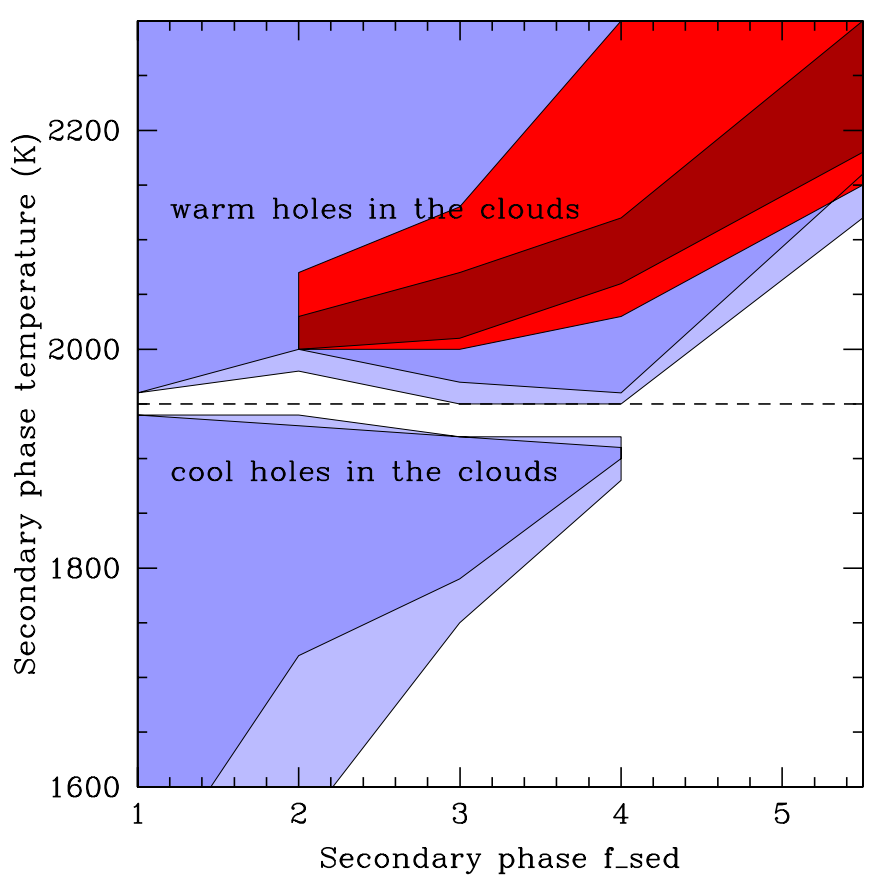

Figure 14. Effective temperatures and cloud sedimentation parameter $\left(f_{\text {sed }}\right)$ values permitted by our data for the secondary phase, provided the primary phase has $f_{\text {sed }}=1$. Cloudiness decreases with increasing $f_{\text {sed }}$. Blue-shaded regions are permitted by the $J /[3.6]$ amplitude ratio observed in our data, while red regions are permitted by the [4.5]/[3.6] amplitude ratio, which turns out to be substantially more constraining. The darker shaded areas are consistent with the data at the $1 \sigma$ level and the lighter areas at $2 \sigma$. The $f_{\text {sed }}$ values probed are $1,2,3,4$, and $\infty$ (corresponding to a completely clear atmosphere). As we cannot extend a plot axis to infinity, for purposes of illustrating the models we have placed the $f_{\text {sed }}=\infty$ results at 5.5 on the $f_{\text {sed }}$ axis. The $T_{\text {eff }}=1950 \mathrm{~K}$ temperature of our primary-phase model is indicated by the dashed horizontal line.

(A color version of this figure is available in the online journal.)

Here, the numerator is simply the flux from the hemisphere where the secondary phase regions appear (which the equation implicitly assumes is the brighter side, provided the amplitude is positive) minus the flux $p$ from the hemisphere uniformly covered by the primary phase. Simplifying and solving for $\epsilon$, we find:

$$
\epsilon=\frac{A_{[3.6]} p}{s-p} .
$$

Our code calculates $\epsilon$ using Equation (6) (with straightforward adjustments to account for the possibility that the hemisphere with only the primary phase will actually be brighter), and then applies Equation (5) to the other bands to predict the amplitude ratios. For a given pair of $f_{\text {sed }}$ values for the primary and secondary phases, we seek values for the temperature of the secondary phase which will simultaneously fit both of our amplitude ratios. We present our results in Figures 14 and 15, making a distinction between models that agree with our data at the $1 \sigma$ and $2 \sigma$ levels.

Figure 14 shows the results if we take the primary phase to have $f_{\text {sed }}=1$. As $f_{\text {sed }}=1$ is the most heavily clouded model, for this model the secondary phase must consist of a region where the cloud is less thick or must involve a change in temperature only. Secondary-phase temperatures can be found that match all our data at the $1 \sigma$ level for $f_{\text {sed }}$ values of $2,3,4$, or $\infty$. The [4.5]/[3.6] amplitude ratio places stronger constraints on the temperature than the $J /[3.6]$ ratio; in fact, almost all models that match [4.5] and [3.6] match our $J$-band results

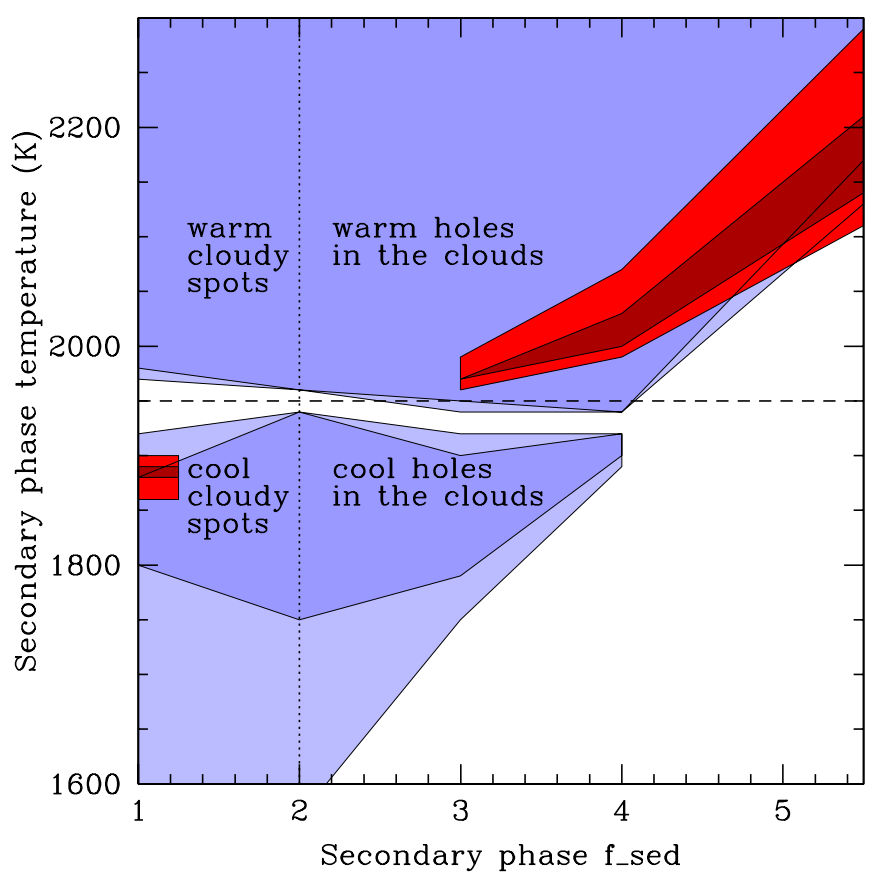

Figure 15. Like Figure 14 but for a primary-phase $f_{\text {sed }}$ value of 2 . The horizontal dashed line corresponds to the $1950 \mathrm{~K} T_{\text {eff }}$ of our primary-phase model. Points above it involve cloud anomalies warmer than the primary phase, while points below it involve anomalies that are cooler. The vertical dotted line at $f_{\text {sed }}=2$ separates secondary-phase models with thicker clouds than the primary phase (left of the line) from those with thinner clouds (right of the line).

(A color version of this figure is available in the online journal.)

as well. As noted above, there is no solution if the secondary phase has the same value of $f_{\text {sed }}$ as the primary phase: cloud inhomogeneities are required to explain the data. For this choice of the primary-phase model, the only solutions correspond to warm "holes" in the clouds: that is, less cloudy patches in the global overcast that also have a higher $T_{\text {eff }}$.

Figure 15 shows the same analysis with a primary-phase $f_{\text {sed }}$ of 2 . Under this model, the secondary phase can have either thicker or thinner clouds than the primary phase. Warm hole-in-the-clouds solutions exist as with our previous model. However, here there is also a small region of the parameter space, permitted at the $1 \sigma$ level, that corresponds to cold regions of especially thick cloud: the signature we might expect if a cool, magnetic starspot has triggered increased condensate formation. A model using a primary-phase $f_{\text {sed }}$ of 3 has the same broad characteristics seen in Figure 15: solutions exist corresponding to either warm "holes" in the clouds or to cold regions of increased cloud thickness.

The full range in permitted values of $\epsilon$ (that is, the projected fraction of DENIS 1058's disk covered by the secondary phase) is $0.8 \%-11.0 \%$ for solutions corresponding to warm "holes" in the clouds and $3 \%-8 \%$ for solutions with cold regions of thicker cloud. In both cases the smaller values of $\epsilon$ correspond to larger differences in $f_{\text {sed }}$ between primary and secondary phases; such scenarios also have the largest $T_{\text {eff }}$ differences and produce the highest brightness contrast. For comparison, Voyager images show the Great Red Spot and its peripheral clouds covering about $3 \%$ of Jupiter's visible disk.

It is worth noting that every scenario permitted by our data has the clearer phase at a higher $T_{\text {eff }}$ than the cloudier phase. This is true whether the clearer phase is the secondary phase (localized, warm "holes" in the clouds) or the primary phase (in which case the secondary phase consists of localized cold 
regions of thicker cloud). It makes sense that we would see down to deeper, warmer layers of the atmosphere in areas of reduced cloud opacity, and this intuition is borne out by atmospheric physical considerations. Well below any cloud decks the global atmospheric temperature everywhere on the brown dwarf must be essentially constant. Compared to the nominal primaryphase model $\left(T_{\text {eff }}=1950 \mathrm{~K}, f_{\text {sed }}=2\right)$, a cloudless model with the same $T_{\text {eff }}$ is roughly $250 \mathrm{~K}$ cooler at depth (pressure $P=10$ bars). Thus if the photosphere of a cloudy $\mathrm{L}$ dwarf were to relax to the thermal profile of a cloudless atmosphere with the same deep atmospheric thermal profile, we would expect that profile to be similar to a cloudless model with a $T_{\text {eff }}$ about $200 \mathrm{~K}$ warmer than the cloudy case and not similar to a cloudless model with the same $T_{\text {eff }}$ as the cloudy case. Thus the finding shown in Figure 15, for example, that the cloudless model that pairs best with an $f_{\text {sed }}=2$ model is $180-260 \mathrm{~K}$ warmer is fully consistent with this picture. The findings that smaller and larger temperature differences are required for the cases of smaller and larger differences in $f_{\text {sed }}$ (respectively) are likewise consistent with the atmospheric thermal profiles. Nothing in our modeling method requires the results to be consistent with this physical reasoning: they simply are. The same pattern has been consistently found in early T dwarfs: Artigau et al. (2009), Radigan et al. (2012), and Apai et al. (2013) all found that their data could be fit only if the temperatures of clearer regions were warmer than those of cloudier regions.

The cloud inhomogeneities we observe could in principle be linked to magnetic phenomena. Magnetic heating in the atmosphere could evaporate condensates and create warm "holes" in the clouds. Similarly, cold regions of thicker cloud could be "cloudy starspots" in which the formation of increased condensates was triggered by a temperature reduction due to the magnetic suppression of convective heat transport. We note that neither mechanism is necessarily required: Jupiter exhibits both cold regions of thick cloud (e.g., the Great Red Spot; Gelino 2002) and warm regions of unusually low cloud opacity, without requiring a magnetic trigger for either.

Formally, the analysis in this section assumes that the $J$-band variability of DENIS 1058 is due to the same set of cloud features as the variability in IRAC ch1, and that the clouds did not change appreciably in the three days between the $J$-band and IRAC observations. The time required for substantial changes in the clouds of $\mathrm{L}$ dwarfs is an open question. However, we note that the strongest constraints come from the IRAC bands, and that the [4.5] data were taken immediately after those at [3.6], rendering it less likely that changing cloud patterns could have affected the measured amplitude ratio.

\subsection{Cold Magnetic Starspots}

Starspots form when locally strong magnetic fields inhibit convection. This can happen only if the convecting gas is sufficiently ionized (i.e., electrically conductive) to interact strongly with the field. It is known based on radio observations that at least some L dwarfs have magnetic fields (Berger 2002; Berger et al. 2005, 2009), but theory indicates that the cool, neutral atmospheres of even early $L$ dwarfs are much too electrically resistive for starspots to form (Mohanty et al. 2002; Gelino et al. 2002; Chabrier \& Küker 2006). In particular, Chabrier \& Küker (2006) comment that the magnetic fields of L dwarfs should allow them to have coronae (which may explain the observed radio emission) but not chromospheres.

Consistent with this theoretical picture, indicators of magnetic activity such as $\mathrm{H} \alpha$ emission and X-ray flux decline as one goes from the mid M-stars down through the L dwarfs (Mohanty et al. 2002; Schmidt et al. 2007). We have already noted that DENIS 1058's $\mathrm{H} \alpha$ emission does not make it an exception to this general trend of decreasing activity in the L dwarfs: its weak emission is not atypical for its spectral type. Also interesting in this context is the fact that the well-known Benz-Güdel relation connecting radio to $\mathrm{X}$-ray flux in radio-emitting late type stars is strongly violated for late $\mathrm{M}$ and cooler objects, such that for $\mathrm{L}$ dwarfs the X-ray fluxes are $\sim 10^{4}$ times too faint relative to the radio (Berger et al. 2005; Stelzer et al. 2012). This may indicate that as temperature decreases, a profound change takes place in the way the magnetic field interacts with the photosphere. If, consistent with theory, we attribute this to the atmosphere becoming uncoupled from the magnetic field, it follows that $\mathrm{L}$ dwarfs cannot exhibit star spots.

However, the fact that $\mathrm{H} \alpha$ emission, though very weak, does still exist for DENIS 1058 (and some other L dwarfs) appears inconsistent with the theoretical arguments that neither starspots nor chromospheres should be able to form. Several suggestions have been put forward to resolve this conundrum. Mohanty et al. (2002) have proposed that buoyant magnetic flux tubes could rise rapidly from ionized regions deep in an L dwarf's interior and release their energy in the object's atmosphere, which would produce $\mathrm{H} \alpha$ emission in the absence of starspots. Alternatively, Lane et al. (2007) attribute their detection of $I$-band variability in the radio-emitting L3.5 dwarf 2MASS J00361617+1821104 to starspots, and get around the neutral-atmosphere problem by proposing magnetic field intensifications across a large enough region that when the field inhibits convection in deep, ionized layers of the star, the effect is still seen at the photosphere. Helling et al. (2011a, 2011b) propose that collisions between dust grains and/or lightning discharges in brown dwarf atmospheres could produce enough ionization to couple the atmosphere to the magnetic field. Under this last scenario, it also seems plausible that the ionization from lightning could be sufficient to explain the observed $\mathrm{H} \alpha$ emission without necessarily being enough to allow starspots.

This plethora of suggestions illustrates that while the interior magnetic dynamos of fully convective objects such as brown dwarfs and very low mass stars have been successfully modeled (Chabrier \& Küker 2006; Dobler et al. 2006), detailed models do not yet exist to constrain photospheric and chromospheric magnetic phenomena in such objects. In the absence of such models we cannot definitively rule out magnetic starspots as an explanation for the variability we have observed in DENIS 1058 - although, as noted above, cloud inhomogeneities are required in addition to starspots to explain our data.

\subsection{Magnetic Emission Regions}

Magnetic fields can produce local emission regions in the form of aurorae, in which energetic electrons flow along external magnetic field lines into an object's atmosphere. Chromospheric emission is produced by magnetic phenomena in denser gas closer to the photosphere, but in some cases is similarly explained by energetic electrons impinging on the gas (see for example the stellar flare model of Allred et al. 2006).

Although stellar flares have too short a characteristic timescale (Berger 2002; Schmidt et al. 2012) to account for the four-hour periodicity we observe in DENIS 1058, either aurorae or lower-level chromospheric emission regions could in principle create rotationally modulated variability. Such variability in magnetically caused emissions has been seen in $\mathrm{L}$ dwarfs. Berger et al. (2009) saw periodic variations in both 
radio and $\mathrm{H} \alpha$ emissions from 2MASSW J0746425+200032. The $\mathrm{H} \alpha$ equivalent width varied from 2.4 to $3.1 \AA$, which corresponds to a variation in the ratio of $\mathrm{H} \alpha$ to bolometric flux of roughly $6.3 \times 10^{-6}$ to $8.2 \times 10^{-6}$. For comparison, $\mathrm{H} \alpha$ emission from DENIS 1058 has been observed at equivalent widths of $1.3 \pm 0.4 \AA, 1.6 \AA$, and $1.0 \pm 0.4 \AA$ by Tinney et al. (1997), Kirkpatrick et al. (1999), and Martín et al. (1999), respectively. Its $\mathrm{H} \alpha$ flux is about $2.1 \times 10^{-6}$ of its bolometric flux (Schmidt et al. 2007).

There are several difficulties with explaining our DENIS 1058 observations in terms of a magnetic emission region. First, the only known marker of magnetic activity in DENIS 1058, the $\mathrm{H} \alpha$ emission, shows no evidence for variability. Although only large $\mathrm{H} \alpha$ variations ( $\gtrsim 50 \%$ ) would have been detected, much larger variations in $\mathrm{H} \alpha$ than in broadband flux are to be expected simply because all of the $\mathrm{H} \alpha$ emission is magnetically generated while most of the IR continuum is not. Second, even supposing the $\mathrm{H} \alpha$ emission to be variable at an undetectable level, the flux variations we observe may be too large relative to the measured $\mathrm{H} \alpha$ flux to be reasonably explained by the same emission regions. Third, granting a scenario in which a very large [3.6] $/ \mathrm{H} \alpha$ emission ratio is possible, it remains difficult to explain the $J$-band variation by the same phenomenon. We expand on these latter two points below.

The IRAC [3.6] magnitude that we measure for DENIS 1058 corresponds to a flux of about $9.9 \times 10^{-16} \mathrm{~W} \mathrm{~m}^{-2}$, and the 2MASS $J$ magnitude of 14.16 corresponds to a flux of $1.1 \times 10^{-15} \mathrm{~W} \mathrm{~m}^{-2}$. These fluxes comprise $9 \%$ and $10 \%$, respectively, of the $1.1 \times 10^{-14} \mathrm{~W} \mathrm{~m}^{-2}$ bolometric flux found by Leggett et al. (2001). The $0.388 \%$ and $0.843 \%$ variations that we observe in these wavelengths therefore correspond to $3.5 \times 10^{-4}$ and $8.5 \times 10^{-4}$ of the bolometric flux, respectively-170 and 400 times larger than the total observed $\mathrm{H} \alpha$ flux. If a localized magnetic emission region is responsible for the variability we observe, its excess luminosity in both IRAC [3.6] and the $J$ band must be more than two orders of magnitude greater than the entire $\mathrm{H} \alpha$ emission from DENIS 1058.

As regards the IRAC [3.6] band, such a luminosity ratio is not necessarily inconsistent with an aurora. Aurorae on the giant planets of our own solar system produce line emission at Ly $\alpha$ (Cook \& Jones 1981; Clarke et al. 1980), H $\alpha$ (Clary \& Hunter 1975; Dyudina et al. 2011), and in the near-infrared emission lines of the $\mathrm{H}_{3}^{+}$molecule. The latter is detectable at $2 \mu \mathrm{m}$ wavelengths (Trafton et al. 1989), but stronger in its fundamental band around $4 \mu \mathrm{m}$ (Oka \& Geballe 1990; Maillard et al. 1990), where its luminosity can indeed be more than two orders of magnitude greater than the entire optical (i.e., $\mathrm{H} \alpha$ ) auroral luminosity (Bhardwaj \& Gladstone 2000).

However, no $J$-band emission lines are observed in the Jovian aurora. Thus, while Jovian-like aurorae on DENIS 1058 might explain its $\mathrm{H} \alpha$ emission and its [3.6] variability, emission lines not seen in such aurorae would be required to explain the even larger variability we see in the $J$-band. Different molecules and different emission lines could exist in the much warmer DENIS 1058, but there are also observational constraints on magnetically caused near-IR emission in warmer objects. Stelzer et al. (2012) obtained simultaneous optical and near-IR spectroscopy of the active M9 dwarf DENIS-P J104814.7-395606 at a time when it was showing emission in the hydrogen Balmer lines out to $n=8$, as well as in the $\mathrm{Ca}$ II $\mathrm{H}$ and $\mathrm{K}$ lines. The circumstances were ideal for detecting emission lines in the $J$ band if any existed, but emission lines were absent not only in the $J$ band but throughout the near-IR, including the $2 \mu \mathrm{m}$ regime where $\mathrm{H}_{3}^{+}$lines are seen in Jupiter. These results for objects bracketing DENIS 1058 in temperature suggest that the $J$-band variability we observe cannot readily be explained in terms of line emission from a magnetically heated region.

None of the difficulties we have outlined above are sufficient to conclusively rule out auroral or chromospheric emission as the cause of DENIS 1058's variability. Although we have shown in Section 6.2 that blackbody continuum emission from a magnetically heated region cannot explain the amplitude ratios we observe, some combination of blackbody and line emission could conceivably do so. It is also possible that an auroral electron beam or other release of magnetic energy could evaporate condensates and create a clearing in the clouds: thus our observations could be due to interactions between magnetic and cloud phenomena. A detailed theory of UCD aurorae, as well as additional observations, is required to constrain such possibilities. At present, we note that a fully self-consistent explanation involving inhomogeneous clouds is possible and seems to involve fewer difficulties than scenarios involving only magnetic emission.

\section{CONCLUSION}

DENIS 1058 exhibits periodic photometric variability in IRAC [3.6] with a period of $4.25_{-0.16}^{+0.26} \mathrm{hr}$ and a peak-to-valley amplitude of $0.388 \% \pm 0.043 \%$. In the $J$-band we measure variations with a larger peak-to-valley amplitude of $0.843 \% \pm$ $0.098 \%$, and a period of $4.31 \pm 0.31 \mathrm{hr}$, which is consistent with our [3.6] results at the $1 \sigma$ level. DENIS 1058 may exhibit very weak IRAC [4.5] variability positively correlated with that at [3.6], but our measured [4.5]/[3.6] amplitude ratio of $0.23 \pm 0.15$ is consistent with zero variability at [4.5].

The different amplitudes we detect at different wavelengths rule out pulsation or tidal distortion due to a close binary as the cause of DENIS 1058's periodic variations; a pulsational interpretation is further ruled out because the period is much too long. This implies that the variability we detect is rotationally modulated. Our photometric period may thus be combined with published $v \sin (i)$ results to obtain a $2 \sigma$ lower limit of $0.111 R_{\odot}$ on DENIS 1058's radius. This implies a mass and age less than $0.055 M_{\odot}$ and $320 \mathrm{Myr}$, respectively, values which are consistent with the young-object kinematics noted by Martín et al. (1997) and Dahn et al. (2002), but not with the lithium non-detections of Martín et al. (1997), Tinney et al. (1997), and Kirkpatrick et al. (1999), nor with the luminosity measurements of Leggett et al. (2001) and Dahn et al. (2002). The age, mass, and radius limits could be reconciled with the luminosity if the effective temperature were $2.7 \sigma$ cooler than measured, though the absence of lithium would remain puzzling. Alternatively, the radius limit would change to a value consistent with the observed luminosity and $\gtrsim 800 \mathrm{Myr}$ age implied by the lithium results if the true rotational velocity were $3.3 \sigma$ less than has been measured. Regardless of which (if either) of these scenarios applies, the large radius limit indicates a near-equatorial viewing geometry: i.e., DENIS 1058's rotation axis is probably inclined substantially more than $45^{\circ}$ to our line of sight.

We have modeled DENIS 1058's variability under the assumption that it is due to cloud inhomogeneities and/or photospheric starspots. Our two-phase cloud models yield viable solutions in which the inhomogeneities take the form of warm "holes" in a global overcast: that is, regions where the clouds are thinner and the $T_{\text {eff }}$ is higher. A smaller number of solutions exist involving regions of even thicker, cooler cloud within the 
global overcast: the scenario we would expect if cold magnetic starspots have triggered the cloud formation. In the absence of cloud inhomogeneities, neither cold nor warm magnetic spots are able to fit our observations. We note that there is not yet a consensus on whether magnetic starspots can occur in L dwarfs.

For every model that can fit our data, the $T_{\text {eff }}$ of the clearer phase is warmer than that of the cloudier phase. Our data cannot be explained by clearings that are colder than the surrounding clouds nor by thicker cloud patches that are warmer than their surroundings. The same result has been found in similar analyses of early T dwarfs by Radigan et al. (2012), Artigau et al. (2009), and Apai et al. (2013). This is consistent with physical considerations regarding the model atmospheres: deep in a brown dwarf's interior, the pressure and temperature under both clearer and cloudier regions must be the same, and if we compare clearer and cloudier model atmospheres with the same deep adiabat, we find that the clearer models invariably have a higher $T_{\text {eff }}$.

Magnetic emission regions in L dwarfs can create variability at radio wavelengths and in the $\mathrm{H} \alpha$ emission line, and in principle could also cause variations at the wavelengths we have observed. Difficulties with such an interpretation include the fact that $\mathrm{H} \alpha$ observations of DENIS 1058 have shown no evidence of variability; that the emission would have to be very efficient in IRAC [3.6] and the $J$-band relative to $\mathrm{H} \alpha$; and that no emission lines capable of explaining the $J$-band variations we observe are readily apparent in spectra of other objects with either auroral or chromospheric emission. None of these difficulties are necessarily fatal, and further observations and theoretical modeling are required to understand L dwarf aurorae. At present, however, explaining the variations of DENIS 1058 by inhomogeneous clouds (which might be coupled to magnetic phenomena) appears to involve the fewest difficulties.

We thank Didier Saumon for supplying us with files containing the model spectra of Saumon \& Marley (2008), which we have used to construct our two-phase models of DENIS 1058. This research was supported by NASA through the Spitzer Exploration Science Program Weather on Other Worlds (program GO 80179) and ADAP award NNX11AB18G. This research is also based on observations obtained at the Southern Astrophysical Research (SOAR) telescope, which is a joint project of the Ministério da Ciência, Tecnologia, e Inovação (MCTI) da República Federativa do Brasil, the U.S. National Optical Astronomy Observatory (NOAO), the University of North Carolina at Chapel Hill (UNC), and Michigan State University (MSU). The SOAR observations reported herein were made under Chilean program CN2012A-055. Radostin Kurtev acknowledges support from Proyecto DIUV23/2009, Centro de Astrofísica de Valparaíso, and FONDECYT through grant 1130140. We thank Nikole Lewis for supplying us with IDL code to measure the noise pixel parameter of our data, for allowing us to read the draft version of her paper describing uses of this parameter in IRAC photometry, and for additional helpful advice. This publication makes use of the SIMBAD online database, operated at CDS, Strasbourg, France, and the VizieR online database (see Ochsenbein et al. 2000). This publication makes use of data products from the Two Micron All Sky Survey, which is a joint project of the University of Massachusetts and the Infrared Processing and Analysis Center/California Institute of Technology, funded by the National Aeronautics and Space Administration and the National Science Foundation. We have also made extensive use of information and code from Press et al. (1992). We have used digitized images from the Palomar Sky Survey (available from http://stdatu.stsci.edu/cgi-bin/dss_form), which were produced at the Space Telescope Science Institute under U.S. Government grant NAG W-2166. The images of these surveys are based on photographic data obtained using the Oschin Schmidt Telescope on Palomar Mountain and the UK Schmidt Telescope.

Facilities: SOAR, Spitzer

\section{APPENDIX \\ ITERATIVE PROCEDURE FOR IMPROVING RELATIVE PHOTOMETRY}

We begin with ordinary relative photometry of DENIS 1058 and the reference stars, constructed using Equation (4) with Stars 7 and 9 dropped from consideration. We normalize the resulting photometry. We then seek to fit a function by which the raw photometry of a given reference star may be divided to remove systematic errors specific to that star and leave only the effects common to all stars. We have already determined that, aside from the nod-position offset, no physically motivated model of systematic errors does as well as a linear function of time. As we are now trying to remove all systematic effects, a function more complex than a linear trend is warranted. We conservatively choose only a quadratic in order to ensure that it cannot mimic a sinusoid by producing two local extrema internal to the data sequence. We seek to model the normalized relative photometry of each reference star using a least-squares SVD fit to a function of time and nod position having the form:

$$
f_{k}(t)=B_{\mathrm{nod}}+A_{0}+A_{1} t+A_{2} t^{2}
$$

where $B_{\text {nod }}$ is zero for images taken in nod position 1 and has a constant value for images taken in nod position 2, and all three parameters will, of course, have unique values for each reference star. We identify outliers more than $2.5 \sigma$ from the fit as bad points. No more than five points are rejected this way in any iteration on any star. Over good points only, we create an adjusted version of the raw photometry for this reference star:

$$
G_{k i}=\frac{F_{k i}}{f_{k}(t)} .
$$

Note that because $f_{k}(t)$ was obtained through a fit to normalized photometry, it is never far from 1.0 for any value of $t$, and therefore $G_{k i}$ will differ only subtly from $F_{k i}$. It should, however, differ in the sense that the systematic errors specific to star $k$ will have been substantially reduced. It remains to get values for $G_{k i}$ for points corresponding to the bad points. Over good points only, we construct relative photometry as follows:

$$
S_{k i}=\frac{G_{k i}}{\sum_{j=2, j \neq k}^{m} F_{j i}} .
$$

Then we replace bad points using:

$$
G_{k b}=\left\langle S_{k i}\right\rangle \sum_{j=2, j \neq k}^{m} F_{j, b}
$$

where $b$ indicates a specific image on which the photometry of star $k$ was bad, and $\left\langle S_{k i}\right\rangle$ is an average over all $i$ where the photometry of star $k$ was good. Effectively, the summed fluxes of all non-deviant stars on image $b$ are being used to create a 
proxy value to replace the deviant photometry of star $k$ on this image. When the photometry of all reference stars has been corrected using Equations (A2) and (A4), we construct a new, second-iteration version of the relative photometry $R_{k i}$ :

$$
R_{k i}=\frac{F_{k i}}{\sum_{j=2, j \neq k}^{m} G_{j i}} .
$$

We proceed with the second iteration, fitting this new relative photometry (after normalization) using Equation (A1). The second and subsequent iterations are the same as the first except that in Equations (A3) and (A4), we can now use the adjusted raw photometry $G_{j i}$ where $F_{j i}$ appeared before. Note, however, that the original raw photometry is always used in the numerator of Equation (A5) to construct the new relative photometry at the start of each iteration. Thus in each iteration, the fit to Equation (A1), and the identification of outliers from this fit, proceeds independently of the fits or outliers found in previous iterations. Points that were found deviant on one iteration may (if the deviance was not intrinsic but was due to as yet uncorrected bad photometry in another star) be accepted on a subsequent iteration, and multiple "layers" of quadratic fits cannot add up to yield, effectively, a fit of much higher order.

\section{REFERENCES}

Ackerman, A., \& Marley, M. 2001, ApJ, 556, 872

Allard, R., Hauschildt, P. H., Alexander, D. R., \& Starrfield, S. 1997, ARA\&A, 35,137

Allard, F., Hauschildt, P. H., Alexander, D. R., Tamanai, A., \& Schweitzer, A. 2001, ApJ, 556, 357

Allred, J. C., Hawley, S. L., Abbett, W. P., \& Carlsson, M. 2006, ApJ, 644, 484 Apai, D., Radigan, J., Buenzli, E., et al. 2013, ApJ, in press (arXiv:1303.4151) Artigau, É, Bouchard, S., Doyon, R., \& Lafrenière, D. 2009, ApJ, 701, 1534 Bailer-Jones, C. A. L., \& Mundt, R. 1999, A\&A, 348, 800

Bailer-Jones, C. A. L., \& Mundt, R. 2001, A\&A, 367, 218

Basri, G., Mohanty, S., Allard, F., et al. 2000, ApJ, 538, 363

Berger, E. 2002, ApJ, 572, 503

Berger, E., Gizis, J. E., Giampapa, M. S., et al. 2008, ApJ, 673, 1080

Berger, E., Rotledge, R. E., Phan-Bao, N., et al. 2009, ApJ, 695, 310

Berger, E., Rutledge, R., Reid, I., et al. 2005, ApJ, 627, 960

Bhardwaj, A., \& Gladstone, G. R. 2000, RvGeo, 38, 295

Buenzli, E., Apai, D., Morley, C., et al. 2012, ApJL, 760, L31

Burgasser, A. J., Looper, D. L., Kirkpatrick, J. D., Cruz, K. L., \& Swift, B. J. 2008, ApJ, 674, 451

Burgasser, A., Marley, M., Ackerman, A., et al. 2002, ApJL, 571, L151

Burrows, A., Marley, M., Hubbard, W. B., et al. 1997, ApJ, 491, 856

Burrows, A., Sudarsky, D., \& Hubeny, I. 2006, ApJ, 640, 1063

Chabrier, G., \& Küker, M. 2006, A\&A, 446, 1027

Charbonneau, D., Allen, L., Megeath, S., et al. 2005, ApJ, 626, 523

Clarke, F. J., Hodgkin, S. T., Oppenheimer, B. R., Robertson, J., \& Haubois, X. 2008, MNRAS, 386, 2009

Clarke, F. J., Oppenheimer, B. R., \& Tinney, C. G. 2002a, MNRAS, 335,1158

Clarke, F. J., Tinney, C. G., \& Covey, K. R. 2002b, MNRAS, 332, 361

Clarke, F. J., Tinney, C. G., \& Hodgkin, S. T. 2003, MNRAS, 341, 239

Clarke, J. T., Moos, H. W., Atreya, S. K., \& Lane, A. L. 1980, ApJL, 241, L179

Clary, R. S., \& Hunter, J. H. 1975, ApJ, 199, 517

Cohen, M., Wheaton, Wm. A., \& Megeath, S. T. 2003, AJ, 126, 1090

Cook, A. F., \& Jones, A. V. 1981, JGR, 86, 8793
Cushing, M. C., Roellig, T. L., Marley, M. S., et al. 2006, ApJ, 648, 614

Dahn, C. C., Harris, H. C., Vrba, F. J., et al. 2002, AJ, 124, 1170

Delfosse, X., Tinney, C., Forveille, T., et al. 1997, A\&A, 327, L25

Deming, D., Fraine, J. D., Sada, P. V., et al. 2012, ApJ, 754, 106

Dobler, W., Stix, M., \& Brandenburg, A. 2006, ApJ, 638, 336

Dyudina, U., Ingersoll, A. P., Wellington, D., Ewald, S. P., \& Porco, C. 2011, EPSC-DPS Conf., 604

Enoch, M. L., Brown, M. E., \& Burgasser, A. J. 2003, AJ, 126, 1006

Fazio, G. G., Hora, J. L., Allen, L. E., et al. 2004, ApJS, 154, 10

Ford, E. 2005, AJ, 129, 1706

Gelino, C. R. 2002, PhD thesis, New Mexico State Univ.

Gelino, C. R., Marley, M. S., \& Holtzman, J. A. 2002, ApJ, 577, 433

Goldman, B. 2005, AN, 326, 1059

Hallinan, G., Bourke, S., Lane, C., et al. 2007, ApJL, 663, L25

Helling, Ch., Jardine, M., \& Mokler, F. 2011a, ApJ, 737, 38

Helling, Ch., Jardine, M., Witte, S., \& Diver, D. A. 2011b, ApJ, 727, 4

Helling, Ch., Jardine, M., Woitke, P., \& Hauschildt, P. H. 2008, ApJL, 675, L105

Kirkpatrick, J. 2005, ARA\&A, 43, 195

Kirkpatrick, J., Reid, I., Liebert, J., et al. 1999, ApJ, 519, 802

Knapp, G. R., Leggett, S. K., Fan, X., et al. 2004, AJ, 127, 3553

Knutson, H., Charbonneau, D., Allen, L., Burrows, A., \& Megeath, S. 2008, ApJ, 673,526

Knutson, H. A., Charbonneau, D., Burrows, A., O'Donovan, F. T., \& Mandushev, G. 2009, ApJ, 691, 886

Koen, C. 2003, MNRAS, 346, 473

Koen, C. 2004, MNRAS, 354, 378

Koen, C. 2005, MNRAS, 360, 1132

Koen, C., Matsunaga, N., \& Menzies, J. 2004, MNRAS, 354, 466

Lane, C., Hallinan, G., Zavala, R. T., et al. 2007, ApJL, 668, L163

Leggett, S., Allard, F., Geballe, T., Hauschildt, P., \& Schweitzer, A. 2001, ApJ, 548, 908

Lewis, N. K., Knutson, H. A., Showman, A. P., et al. 2013, ApJ, 766, 95

Littlefair, S. P., Dhillon, V. S., Marsh, T. R., et al. 2008, MNRAS, 391, L88

Loh, E., Beil, J., Davis, M., et al. 2012, PASP, 124, 343

Looper, D. L., Kirkpatrick, J. D., Cutri, R. M., et al. 2008, ApJ, 686, 528

Maillard, J.-P., Drossart, P., Watson, J., Kim, S., \& Caldwell, J. 1990, ApJL, 363, L37

Marley, M. S., Lunine, J. I., \& Hubbard, W. B. 1990, ApJL, 348, L37

Marley, M. S., Saumon, D., \& Goldblatt, C. 2010, ApJ, 723, 117

Martín, E., Basri, G., Delfosse, X., \& Forveille, T. 1997, A\&A, 327, L29

Martín, E. L., Delfosse, X., Basri, G., et al. 1999, AJ, 118, 2466

Mohanty, S., Basri, G., Shu, F., Allard, F., \& Chabrier, G. 2002, ApJ, 571, 469

Morales-Calderón, M., Stauffer, J. R., Kirkpatrick, J. D., et al. 2006, ApJ, 653,1454

Ochsenbein, F., Bauer, P., \& Marcout, J. 2000, ApJS, 143, 23

Oka, T., \& Geballe, T. 1990, ApJL, 351, L53

Press, W. H., Teukolsky, S. A., Vetterling, W. T., \& Flannery, B. P. 1992, Numerical Recipes in C (2nd ed.; New York: Cambridge Univ. Press)

Radigan, J., Jayawardhana, R., Lafrenière, D., et al. 2012, ApJ, 750, 105

Reach, W., Megeath, S., Cohen, M., et al. 2005, PASP, 117, 978

Reid, I. N., Cruz, K. L., Burgasser, A. J., \& Liu, M. C. 2008, AJ, 135, 580

Saumon, D., \& Marley, M. 2008, ApJ, 689, 1327

Schmidt, S., Cruz, K., Bongiorno, B., Liebert, J., \& Reid, I. 2007, AJ, 133, 2258

Schmidt, S., Kowalski, A., Hawley, S., et al. 2012, ApJ, 745, 14

Skrutskie, M., Cutri, R. M., Stiening, R., et al. 2006, AJ, 131, 1163

Stelzer, B., Alcala, J, Biazzo, K., Ercolano, B., et al. 2012, A\&A, 537, A94

Stephens, D., Leggett, S., Cushing, M., et al. 2009, ApJ, 702, 154

Tinney, C., Delfosse, X., \& Forveille, T. 1997, ApJL, 490, L95

Tinney, C. G., Burgasser, A. J., \& Kirkpatrick, J. D. 2003, ApJ, 126, 975

Todorov, K. O., Deming, D., Knutson, H. A., et al. 2012, ApJ, 746, 111

Trafton, L., Lester, D., \& Thompson, K. 1989, ApJL, 343, L73

Tsuji, T., Ohnaka, K., \& Aoki, W. 1996, A\&A, 305, L1

van Dokkum, P. 2001, PASP, 113, 1420

Vrba, F. J., Henden, A. A., Luginbuhl, C. B., et al. 2004, AJ, 127, 2948 